\title{
Symmetry Properties and Explicit Solutions of the Generalized Weierstrass System
}

\author{
P. Bracken* \\ A. M. Grundland ${ }^{\dagger}$
}

CRM-2673

March 2000

*Centre de Recherches Mathématiques, Université de Montréal, 2920 Chemin de la Tour, Pavillon André Aisenstadt, C. P. 6128 Succ. Centre Ville, Montréal, QC, H3C 3J7 Canada.bracken@CRM. UMontreal.ca

†Centre de Recherches Mathématiques, Université de Montréal, 2920 Chemin de la Tour, Pavillon André Aisenstadt, C. P. 6128 Succ. Centre Ville, Montréal, QC, H3C 3J7 Canada.grundlan@CRM.UMontreal.ca 



\begin{abstract}
The method of symmetry reduction is systematically applied to derive several classes of invariant solutions for the generalized Weierstrass system inducing constant mean curvature surfaces and to the associated two-dimensional nonlinear sigma model. A classification of subgroups with generic orbits of codimension one of the Lie point symmetry group for these systems provides a tool for introducing symmetry variables and reduces the initial systems to different nonequivalent systems of ordinary differential equations. We perform a singularity analysis for them in order to establish whether these ordinary differential equations have the Painlevé property. These ordinary differential equations can then be transformed to standard forms and next solved in terms of elementary and Jacobi elliptic functions. This results in a large number of new solutions and in some cases new interesting constant mean curvature surfaces are found. Furthermore, this symmetry analysis is extended to include conditional symmetries by subjecting the original systems to certain differential constraints. In this case, several types of nonsplitting algebraic, trigonometric and hyperbolic multi-soliton solutions have been obtained in explicit form. A new procedure for constructing solutions of the overdetermined system which is composed of the generalized Weierstrass system and the complex eikonal equations is studied. Finally, an approach to the classical configurations of strings in three-dimensional Euclidean space based on the obtained solutions of the generalized Weierstrass system is presented.
\end{abstract}

\title{
Résumé
}

La méthode de réduction par symétries est appliquée systématiquement pour dériver plusieurs classes de solutions invariantes du système de Weierstrass généralisé induisant des surfaces de courbure moyenne constante et du modèle sigma euclidien bidimensionnel associé à ce système. Une classification des sous-groupes avec orbites génériques de codimension un des groupes de Lie de symétrie ponctuels pour ces systèmes fournit un moyen d'introduire des variables de symétrie et réduit le système initial à différents systèmes inéquivalents d'équations différentielles ordinaires. Nous effectuons une analyse des singularités afin d'établir si ces équations différentielles ordinaires possèdent la propriété de Painlevé. Ces équations différentielles ordinaires peuvent alors être transformées dans des formes standards et ensuite résolues en termes de fonctions élémentaires et de fonctions elliptiques de Jacobi. Cela résulte en un grand nombre de nouvelles solutions et, dans certains cas, de nouvelles surfaces à courbure moyenne constante sont trouvées. De plus, cette analyse des symétries est étendue au cas des symétries conditionnelles en soumettant les systèmes initiaux à certaines contraintes différentielles. Dans ce cas, plusieurs solutions multi-solitoniques non-séparantes de type algébrique, trigonométrique et elliptique sont obtenues sous une forme explicite. Une nouvelle procédure pour construire des solutions du système surdéterminé composé du système de Weierstrass généralisé et des équations eikonales complexes est étudiée. Finalement, nous présentons une nouvelle application des configurations classiques des cordes dans l'espace euclidien tridimensionnel basées sur les solutions obtenues des systèmes de Weierstrass généralisés. 



\section{Contents}

1. Introduction.

2. The Symmetry Group, its Subalgebras and their Reductions to ODEs.

3. The Second Order System.

4. Group Invariant Solutions.

5. The Non-Splitting Solutions of the Generalized Weierstrass System.

6. Differential Constraints and Solutions of the GW System.

7. The Complex Laplace and Eikonal Differential Constraints.

8. Application to Classical String Theory.

9. Summary and Concluding Remarks.

10. Appendix A1.

11. Tables. 


\section{INTRODUCTION}

The expressions describing minimal surfaces imbedded in three-dimensional Euclidean space were first formulated by A. Enneper and K. Weierstrass $[1,2]$ one and a half centuries ago. They start by introducing two holomorphic functions $\psi(z), \phi(z)$ and three complex valued functions $\omega_{1}$, $\omega_{2}$ and $\omega_{3}$ which satisfy the following system of equations

$$
\partial \omega_{1}=i\left(\psi^{2}+\phi^{2}\right), \quad \partial \omega_{2}=\psi^{2}-\phi^{2}, \quad \partial \omega_{3}=-2 \psi \phi, \quad \bar{\partial} \psi=0, \quad \bar{\partial} \phi=0,
$$

where the derivatives are abbreviated $\partial=\partial / \partial z$ and $\bar{\partial}=\partial / \partial \bar{z}$. The bar denotes the complex conjugate. They show that if the system of three real-valued functions $X_{i}(z, \bar{z}), i=1,2,3$ are considered as a coordinate system for a surface immersed in $\mathbb{R}^{3}$, defined as follows

$$
\begin{gathered}
X_{1}=\operatorname{Re} \omega_{1}=\operatorname{Re} \int_{C} i\left(\psi^{2}+\phi^{2}\right) d z, \\
X_{2}=\operatorname{Re} \omega_{2}=\operatorname{Re} \int_{C}\left(\psi^{2}-\phi^{2}\right) d z, \\
X_{3}=\operatorname{Re} \omega_{3}=-\operatorname{Re} \int_{C} 2 \psi \phi d z,
\end{gathered}
$$

(where $C$ is any contour in the domain of common holomorphicity of both functions $\psi$ and $\phi$ ), then the functions $X_{i}(z, \bar{z})$ determine a minimal surface. The minimal lines on this surface are given by the parametric lines $z=$ constant and $\bar{z}=$ constant, respectively.

More recently, this idea was substantially generalized by B. Konopelchenko [3] who established the connection between certain classes of constant mean curvature surfaces and the trajectories of an infinite-dimensional Hamiltonian system. Namely, he considered the nonlinear Dirac-type system of equations for two complex valued functions $\psi_{1}$ and $\psi_{2}$ given by

$$
\begin{array}{ll}
\partial \psi_{1}=p \psi_{2}, & \bar{\partial} \psi_{2}=-p \psi_{1}, \\
\bar{\partial} \bar{\psi}_{1}=p \bar{\psi}_{2}, & \partial \bar{\psi}_{2}=-p \bar{\psi}_{1}, \\
\multicolumn{2}{c}{p=\left|\psi_{1}\right|^{2}+\left|\psi_{2}\right|^{2} .}
\end{array}
$$

Then he proved that the real valued functions $X_{i}(z, \bar{z}), i=1,2,3$,

$$
\begin{aligned}
& X_{1}+i X_{2}=2 i \int_{\gamma}\left(\bar{\psi}_{1}^{2} d z^{\prime}-\bar{\psi}_{2}^{2} d \bar{z}^{\prime}\right), \\
& X_{1}-i X_{2}=2 i \int_{\gamma}\left(\psi_{2}^{2} d z^{\prime}-\psi_{1}^{2} d \bar{z}^{\prime}\right), \\
& X_{3}=-2 \int_{\gamma}\left(\bar{\psi}_{1} \psi_{2} d z^{\prime}+\psi_{1} \bar{\psi}_{2} d \bar{z}^{\prime}\right),
\end{aligned}
$$

define constant mean curvature surfaces imbedded in $\mathbb{R}^{3}$. On account of the system (1.3), the right hand side of (1.4) does not depend on the choice of contour $\gamma$ in $\mathbb{C}$. The Gaussian curvature and first fundamental form on the surface are given by [4]

$$
K=-\frac{\partial \bar{\partial}(\ln p)}{p^{2}}, \quad \Omega=4 p^{2} d z d \bar{z},
$$


in isothermic coordinates. Konopelchenko called the modified version (1.3) of Weierstrass-Enneper system (1.1) the generalized Weierstrass (GW) system. These formulae are the starting point for the symmetry analysis in this paper, and we will refer to it as such. The theory of constant mean curvature surfaces has had a great impact on many problems with physical applications. In particular, to such diverse areas as in the field of two-dimensional gravity $[\mathbf{5 , 6}]$, quantum field theory $[5,7]$, statistical physics $[\mathbf{8 , 9}]$ and fluid dynamics $[\mathbf{1 0 , 1 1}]$. It is worth mentioning an application of recent interest, namely, the propagation of a string through space-time [12]. It describes a surface called its world sheet. When one quantizes a string, the result is an ordinary two-dimensional point particle quantum field theory on a given surface. Thus, one can say that first quantized string theory is the study of conformal field theories on Riemann surfaces. Another relevant application of recent interest is in the area of statistical mechanics. Any two-dimensional statistical system near a second order phase transition can be described by a conformally invariant theory [8]. Near a phase transition, fluctuations of the fields are correlated over very long length scales and appear on all scales essentially equally. Since no scale is preferred, it becomes scale or conformally invariant. Thus, the specific lattice becomes relatively unimportant, and so a type of universal behavior is displayed.

The system of equations (1.3) are integrable nonlinear PDEs, since they are characterized by being the compatibility condition between two associated linear PDEs, the Lax pair, for any two component wave function [13] given by

$$
\partial \Phi=\frac{2}{\mu+1} M \Phi, \quad \bar{\partial} \Phi=\frac{2}{\mu-1} M^{\dagger} \Phi
$$

where $\mu$ is the spectral parameter and the $2 \times 2$ matrix $M$ is given by

$$
M=A+\frac{J}{p^{2}} A^{\dagger}, \quad \operatorname{det} M=-\frac{2 J}{p^{2}}, \quad \bar{\partial} J=0 .
$$

Here, $M^{\dagger}$ is the Hermitian conjugate of the matrix $M$, the conserved quantity

$$
J=\bar{\psi}_{1} \partial \psi_{2}-\psi_{2} \partial \bar{\psi}_{1}, \quad \bar{\partial} J=0
$$

is the current for the system (1.3), and $A$ is a degenerate nilpotent matrix which can be decomposed as follows

$$
A=-\bar{\psi}_{1} \psi_{2} \sigma_{3}-\bar{\psi}_{1}^{2} \sigma_{+}+\psi_{2}^{2} \sigma_{-}, \quad \sigma_{ \pm}=\frac{1}{2}\left(\sigma_{1} \pm i \sigma_{2}\right)
$$

where $\sigma_{1}, \sigma_{2}, \sigma_{3}$ are Pauli matrices

$$
\sigma_{1}=\left(\begin{array}{cc}
0 & 1 \\
1 & 0
\end{array}\right), \quad \sigma_{2}=\left(\begin{array}{cc}
0 & -i \\
i & 0
\end{array}\right), \quad \sigma_{3}=\left(\begin{array}{cc}
1 & 0 \\
0 & -1
\end{array}\right) .
$$

It has been demonstrated [14] that GW system (1.3) can be decoupled into a direct sum of elliptic Sh-Gordon and Laplace equations. If we change the dependent variables $\psi_{1}$ and $\psi_{2}$ in (1.3) to the new dependent variables $p$ and $J$, then the GW system (1.3) can be written in the equivalent form

$$
\partial \bar{\partial} \ln p=\frac{|J|^{2}}{p^{2}}-p^{2}, \quad \bar{\partial} J=0 .
$$

It was shown using the conditional symmetry method [15], that the GW system admits an AutoBäcklund transformation for any holomorphic function $J$,

$$
\begin{array}{cl}
\partial p=-\lambda q p^{2}+\frac{\partial q}{q} p-\frac{\lambda J}{q}, & \bar{\partial} J=0 \\
\bar{\partial} p=-\frac{\bar{J}}{\lambda q} p^{2}-\frac{\bar{\partial} q}{q} p-\frac{q}{\lambda}, \quad \lambda \in \mathbb{C}
\end{array}
$$


where the function $q$ satisfies the elliptic Sh-Gordon equation (1.8). The arbitrary complex constant $\lambda$ is the Bäcklund parameter. The compatibility condition for (1.9) reproduces the system (1.8) in the variable $q$. It has also been shown [15], that for a chosen solution $q$ of (1.8), the symmetry group $G$ of the overdetermined system (1.8) and (1.9) with two-dimensional orbits has a complete set of two functionally independent invariants. Thus, the solution $p$ of the initial equations (1.8) and (1.9) can be expressed in terms of these invariants. In other words, they are invariant under the finite Abelian Lie algebra given by

$$
\begin{aligned}
& Z_{1}=\partial-\left(\frac{\lambda J}{q}-\frac{\partial q}{q} p+\lambda q p^{2}\right) \partial_{p}, \quad \bar{\partial} J=0 \\
& Z_{2}=\bar{\partial}-\left(\frac{q}{\lambda}+\frac{\bar{\partial} q}{q} p+\frac{\bar{J}}{\lambda q} p^{2}\right) \partial_{p},
\end{aligned}
$$

where these vector fields are parametrized by the function $q$ which satisfies (1.8), and constant $\lambda \in \mathbb{C}$. The difference between the classical symmetry reduction and the conditional one [16] is that the group associated with a classical approach to system (1.8) maps all solutions into other solutions of the same system, whereas for a conditional symmetry method, the associated group $G$ maps only a subset of solutions of system (1.8) into solutions of the overdetermined system, that is, the original system (1.8) subjected to given differential constraints (1.9).

Furthermore, by linearizing the Ricatti system (1.9), that is, by taking the homogeneous coordinates $\phi_{1}$ and $\phi_{2}$ in (1.9) such that $p=\phi_{1} / \phi_{2}$, we obtain, for any holomorphic function $J$, the associated linear spectral problem for (1.8)

$$
\partial\left(\begin{array}{c}
\phi_{1} \\
\phi_{2}
\end{array}\right)=\left(\begin{array}{cc}
\frac{\partial q}{2 q} & -\frac{\mu J}{q} \\
\mu q & -\frac{\partial q}{2 q}
\end{array}\right)\left(\begin{array}{c}
\phi_{1} \\
\phi_{2}
\end{array}\right), \quad \bar{\partial}\left(\begin{array}{c}
\phi_{1} \\
\phi_{2}
\end{array}\right)=\left(\begin{array}{cc}
-\frac{\bar{\partial} q}{2 q} & -\frac{q}{\mu} \\
\bar{J} & \bar{\partial} q \\
\overline{\mu q} & \frac{\overline{2}}{2 q}
\end{array}\right)\left(\begin{array}{c}
\phi_{1} \\
\phi_{2}
\end{array}\right), \quad \bar{\partial} J=0 .
$$

After the change of variables from $p$ and $J$ to the variables $\psi_{1}$ and $\psi_{2}$, the Lax pair (1.11) is equivalent to (1.6). The permutability theorem for the Auto-Bäcklund transformation for system (1.3) has recently been formulated by the authors [15] and new classes of nonsplitting multi-soliton solutions of system (1.3) have been obtained.

The objective of this paper is a systematic analysis of GW system (1.3) from the symmetry group point of view. We focus on constructing several classes of solutions obtained from the symmetry reduction method $[\mathbf{1 7}, \mathbf{1 8}]$. The extension of the classical Lie approach to group invariant solutions of (1.3) admitting different differential constraints (DCs) has been incorporated. Each of the solutions presented provides us with different classes of constant mean curvature surface imbedded in threedimensional Euclidean space.

This paper is organized as follows. Section 2 is devoted to the construction of Lie point symmetries and their subalgebras for GW system (1.3). The reductions of (1.3) to ODEs requires consideration of 3-dimensional subalgebras. The general integrals of these ODEs are presented.

In Section 3, we investigate the connection between GW system (1.3) and a two-dimensional nonlinear sigma model. This link together with conservation laws for (1.3) allows us to establish several useful transformations which simplify the structure of GW system (1.3). Section 4 deals with the associated sigma model. The symmetry algebra, list of representatives of conjugacy classes of subalgebras, the corresponding invariants and reduced differential equations are given. Solutions of of GW system (1.3) are provided by means of a number of reduced equations of the associated 
sigma model. In some cases, solitary and doubly periodic solutions of GW system in terms of Jacobi elliptic functions are obtained.

Section 5 contains the nonsplitting solutions of GW system (1.3) of a number of reduced equations and solutions invariant under the scaling transformations. Several Propositions have been formulated which are useful for constucting several classes of solutions. Section 6 discusses in detail a set of DCs which allow GW system (1.3) to become a decoupled linear system of equations, and we solve these in terms of Bessel functions of zero order. In Section 7, we look for conditions necessary for solvability of an overdetermined system composed of GW system (1.3) and complex eikonal equations, and explicit solutions are given. In Section 8, an interesting application to the area of string theory of our solutions obtained here is presented.

2 THE SYMMETRY GROUP, ITS SUBALGEBRAS AND THEIR REDUCTIONS TO ODES.

The symmetry group of GW system (1.3) can be computed using the appropriate MACSYMA program [19]. This provides us with a set of determining equations from which we can find the symmetry algebra $L$ of infinitesimal symmetries of system (1.3). It is spanned by the vector fields

$$
\begin{gathered}
T_{1}=\partial, \quad T_{2}=\bar{\partial} \\
D_{1}=z \partial-\frac{1}{2}\left(\bar{\psi}_{1} \partial_{\bar{\psi}_{1}}+\psi_{2} \partial_{\psi_{2}}\right), \\
D_{2}=\bar{z} \bar{\partial}-\frac{1}{2}\left(\psi_{1} \partial_{\psi_{1}}+\bar{\psi}_{2} \partial_{\bar{\psi}_{2}}\right), \\
K_{1}=z^{2} \partial-z\left(\bar{\psi}_{1} \partial_{\bar{\psi}_{1}}+\psi_{2} \partial_{\psi_{2}}\right), \\
K_{2}=\bar{z}^{2} \bar{\partial}-\bar{z}\left(\psi_{1} \partial_{\psi_{1}}+\bar{\psi}_{2} \partial_{\bar{\psi}_{2}}\right), \\
H=\psi_{1} \partial_{\psi_{1}}-\bar{\psi}_{1} \partial_{\bar{\psi}_{1}}+\psi_{2} \partial_{\psi_{2}}-\bar{\psi}_{2} \partial_{\bar{\psi}_{2}} .
\end{gathered}
$$

The physical interpretation of the Lie algebra $L$ is as follows. Here, $T_{1}$ and $T_{2}$ generate translations in the $z$ and $\bar{z}$ directions, respectively. The generators $D_{1}$ and $D_{2}$ correspond to two different types of dilations and the generators $K_{1}$ and $K_{2}$ represent two types of conformal transformations. The generator $H$ reflects the fact that the GW system is invariant under phase transformations. To show this, it is convenient to use polar coordinates for the functions $\psi_{i}$, that is to put

$$
\psi_{i}=R_{i} e^{i \alpha_{i}}
$$

and then write the generator $H$ in terms of $R_{i}$ and $\alpha_{i}$. In this case, the field $H$ takes the simple form

$$
H=-i\left(\partial_{\alpha_{1}}+\partial_{\alpha_{2}}\right)
$$

The commutation relations for this algebra are given in Table 1. We see that the Lie algebra can be decomposed into a direct sum of two $\operatorname{sl}(2, \mathbb{C})$ subalgebras in addition with generator $H$. The vector field $H$ represents the center of the Lie algebra $L$. Thus we find that

$$
L=\{\operatorname{sl}(2, \mathbb{C})\}_{1} \oplus\{\operatorname{sl}(2, \mathbb{C})\}_{2} \oplus H .
$$

where the subalgebras $\{s l(2, \mathbb{C})\}_{i}$ are spanned by $\left\{T_{i}, D_{i}, K_{i}\right\}, i=1,2$, respectively. Subalgebras of the direct sum $\{s l(2, \mathbb{C})\}_{1} \oplus\{\operatorname{sl}(2, \mathbb{C})\}_{2}$ can be classified by using an adaptation of the Goursat twist method to Lie algebras $[20,21]$. Note that the vector fields $K_{i}$ can be rectified by a point transformation involving independent and dependent variables in such a way that $K_{i}$ is equivalent to $T_{i}$. This fact simplifies considerably the classification of subalgebras of $L$. 
The results for representatives of the conjugacy classes of one-dimensional subalgebras of the symmetry algebra $L$ are listed in Table 2 using a standard method [22]. The discrete transformations of GW system (1.3) were used in order to restrict the range of parameters appearing in the classification. The proposition is that any one-dimensional subalgebra of $L$ is conjugate under the action of the group $G$ to precisely one algebra $L_{j}, 1 \leq j \leq 7$ in the list. No two members occuring in the list are mutually conjugate. The list of invariants of the corresponding Lie subalgebras and also the corresponding reduced ODEs are given in Table 2. The functions $A, B, C, D$ and $\xi$ denote the five invariants for the given subalgebras $L_{j}$, where $\xi$ is the symmetry variable. The main feature of the symmetry reduction method is that the set of symmetry variables enables us to reduce, after some transformations, the original system of PDEs to sets of four coupled ODEs. These ODEs can very often be explicitly integrated in terms of known functions or at least their singularity structure can be investigated using the Painlevé test [23]. It is shown in many cases that the result of the test is positive for the reduced systems of ODEs. After some change of variables, these ODEs can be written in closed analytic form which can be decoupled and solved in many cases. The Lie algebra obtained from the infinitesimal symmetries of the GW system (1.3) listed in Table 2 have to obey the restriction coming from the requirement that the quantities $z$ and $\bar{z}$ and as well $\psi_{i}$ and $\bar{\psi}_{i}$ cannot be viewed as independent variables in $\mathbb{C} \times \mathbb{C}$, respectively, as demanded by symmetry criterion. This fact is a strong limitation on the admitted class of invariant solutions of (1.3). Thus if we interpret $z$ and $\bar{z}$ as coordinates in the complex plane $\mathbb{C}$, and $\psi_{i}$ and $\bar{\psi}_{i}$ as complex conjugate valued functions on $\mathbb{C}$, then the following is a list of representatives of conjugacy classes of one-dimensional subalgebras, the corresponding invariants, and their associated reductions and solutions of (1.3). The four invariants $A, B, C, D$ depend on the argument $\xi$. Here, we only summarize the results.

1. $L_{1}=\left\{D_{1}-D_{2}+\beta H\right\}, \beta \in \mathbb{R}, \xi=|z|^{2}$.

$$
\begin{array}{ll}
\psi_{1}=A(\xi) z^{\beta+1 / 2}, & \bar{\psi}_{1}=\bar{A}(\bar{\xi}) \bar{z}^{\beta+1 / 2}, \\
\psi_{2}=B(\xi) z^{\beta-1 / 2}, & \bar{\psi}_{2}=\bar{B}(\xi) \bar{z}^{\bar{\beta}-1 / 2} .
\end{array}
$$

The corresponding reduced differential equations are

$$
\begin{gathered}
\dot{A}+\frac{2 \beta+1}{2 \xi} A-\left(|A|^{2} \xi^{\beta-1 / 2}+|B|^{2} \xi^{\beta-3 / 2}\right) B=0, \\
\dot{B}+\left(|A|^{2} \xi^{\beta+1 / 2}+|B|^{2} \xi^{\beta-1 / 2}\right) A=0 .
\end{gathered}
$$

The simplest solution of equations (2.4) which corresponds to an analytic choice of the functions $\psi_{i}$ is given by

$$
\psi_{1}=\lambda \bar{a}^{1 / 2}(z \bar{z})^{a / 2}, \quad \psi_{2}=\lambda a^{1 / 2}(z \bar{z})^{(a-1) / 2} \bar{z},
$$

where $\lambda$ and $a$ are complex constants such that $a+\bar{a}=1$ and $|\lambda|^{4}=1 / 16$. The associated constant mean curvature surface can be computed from equations (1.4), namely,

$$
X_{1}-i X_{2}=4 i \lambda^{2} z^{a} \bar{z}^{a+1}, \quad X_{1}+i X_{2}=-4 i \bar{\lambda}^{2} \bar{z}^{\bar{a}} z^{\bar{a}+1}, \quad X_{3}=-\frac{1}{2} \ln \left(z^{a} \bar{z}^{\bar{a}}\right) .
$$

Solving the above system, we get that

$$
X_{1}^{2}+X_{2}^{2}=1, \quad z^{a} \bar{z}^{\bar{a}}=e^{-2 X_{3}},
$$

which describes a cylinder with unit radius and having $X_{3}$ as symmetry axis. The Gaussian curvature is $K=1$. 
2. $L_{2}=\left\{T_{2}+D_{1}+\beta H\right\}, \beta \in \mathbb{C}$ case leads to trivial constant solutions $\psi_{i}$ of the GW system $(1.3)$.

3. $L_{3}=\left\{T_{1}+T_{2}+\epsilon H\right\}, \epsilon= \pm 1, \xi=z-\bar{z}$ and

$$
\begin{array}{ll}
\psi_{1}=A(\xi) e^{i \epsilon z}, & \bar{\psi}_{1}=\bar{A}(\bar{\xi}) e^{-i \epsilon \bar{z}} \\
\psi_{2}=B(\xi) e^{i \epsilon z}, & \bar{\psi}_{2}=\bar{B}(\bar{\xi}) e^{-i \epsilon \bar{z}} .
\end{array}
$$

The reduction to differential equations is given by

$$
\dot{A}+i \epsilon A=\left(|A|^{2}+|B|^{2}\right) B, \quad \dot{B}=\left(|A|^{2}+|B|^{2}\right) A .
$$

The explicit solution of equations (2.5) has the form

$$
\psi_{1}=\lambda(a-i \epsilon)^{1 / 2} \exp (a(z-\bar{z}) / 2+i \epsilon(z+\bar{z}) / 2), \psi_{2}=\lambda(a+i \epsilon)^{1 / 2} \exp (a(z-\bar{z}) / 2+i \epsilon(z+\bar{z}) / 2),
$$

where $\epsilon= \pm 1, a \in \mathbb{R}$, and $|\lambda|^{4}=1 / 16$. The corresponding constant mean curvature surface is determined by relations (1.4)

$$
X_{1}^{2}+X_{2}^{2}=1, \quad X_{3}=-\mathbb{R} e((a+i \epsilon) z)
$$

which describe a cylinder with unit radius and symmetry axis $X_{3}$. The Gaussian curvature is $K=1$.

4. $L_{4}=\left\{D_{1}+\beta H\right\}, \xi=\bar{z}$ and

$$
\begin{array}{ll}
\psi_{1}=A z^{\beta} \bar{z}^{-\beta+1 / 2}, & \bar{\psi}_{1}=\bar{A} z^{-\beta-1 / 2} \bar{z}^{\bar{\beta}}, \\
\psi_{2}=B \bar{z}^{-\bar{\beta}} z^{\beta-1 / 2}, & \bar{\psi}_{2}=\bar{B} z^{-\beta} \bar{z}^{\beta-1 / 2} .
\end{array}
$$

The corresponding reduced system of equations is algebraic

$$
\beta A=\left(|A|^{2}+|B|^{2}\right) B, \quad \beta B=\left(|A|^{2}+|B|^{2}\right) A
$$

A general solution of these equations can be written in the following form

$$
\begin{aligned}
& \psi_{1}=\sqrt{\frac{\beta}{2}} e^{i \theta} z^{\beta} \bar{z}^{-\beta-1 / 2}, \quad \bar{\psi}_{1}=\sqrt{\frac{\beta}{2}} e^{-i \theta} z^{-\beta-1 / 2} \bar{z}^{\beta}, \\
& \psi_{2}=\sqrt{\frac{\beta}{2}} e^{i \theta} z^{\beta-1 / 2} \bar{z}^{-\beta}, \quad \bar{\psi}_{2}=\sqrt{\frac{\beta}{2}} e^{-i \theta} z^{-\beta} \bar{z}^{\beta-1 / 2} .
\end{aligned}
$$

The corresponding mean curvature surface is given by

$$
X_{1}^{2}+X_{2}^{2}=\frac{1}{4}, \quad X_{3}=-2 \beta \ln (|z|),
$$

which is again a cylinder of radius $1 / 2$.

Similar results are obtained for the case of the subalgebra $L_{6}=D_{2}+\beta H$ when $z$ is replaced by $\bar{z}$ and $\left(\psi_{1}, \bar{\psi}_{1}, \psi_{2}, \bar{\psi}_{2}\right)$ is replaced by $\left(\psi_{2}, \bar{\psi}_{2}, \psi_{1}, \bar{\psi}_{1}\right)$, respectively.

5. $L_{5}=\left\{T_{1}+\epsilon H\right\}, \epsilon= \pm 1, \xi=\bar{z}$ with

$$
\begin{array}{ll}
\psi_{1}=A_{0} e^{\epsilon(z-\bar{z})}, & \bar{\psi}_{1}=\bar{A}_{0} e^{-\epsilon(z-\bar{z})}, \\
\psi_{2}=B_{0} e^{\epsilon(z-\bar{z})}, & \bar{\psi}_{2}=\bar{B}_{0} e^{-\epsilon(z-\bar{z})},
\end{array}
$$


where $A_{0}, B_{0} \in \mathbb{C}, \epsilon= \pm 1$. The reduction is an algebraic system of equations

$$
\epsilon A_{0}=\left(\left|A_{0}\right|^{2}+\left|B_{0}\right|^{2}\right) B_{0}, \quad \epsilon B_{0}=\left(\left|A_{0}\right|^{2}+\left|B_{0}\right|^{2}\right) A_{0} .
$$

The general solution is given by

$$
\begin{aligned}
& \psi_{1}=\frac{e^{i \theta}}{\sqrt{2}} e^{\epsilon(z-\bar{z})}, \quad \bar{\psi}_{1}=\frac{e^{-i \theta}}{\sqrt{2}} e^{-\epsilon(z-\bar{z})}, \quad \epsilon= \pm 1 \\
& \psi_{2}=\frac{e^{i \theta}}{\sqrt{2}} e^{\epsilon(z-\bar{z})}, \quad \bar{\psi}_{2}=\frac{e^{-i \theta}}{\sqrt{2}} e^{-\epsilon(z-\bar{z})},
\end{aligned}
$$

and the surface is again a cylinder of radius 1 and the z-axis as symmetry axis. Similar results are obtained to the ones obtained above for the subalgebra $L_{7}=T_{2}+\epsilon H$ if $\bar{z}$ is replaced by $z$ and $\left(\psi_{1}, \bar{\psi}_{1}, \psi_{2}, \bar{\psi}_{2}\right)$ is replaced by $\left(\bar{\psi}_{1}, \psi_{1}, \bar{\psi}_{2}, \psi_{2}\right)$, respectively.

We classify two-dimensional subalgebras of the symmetry algebra (2.1) into conjugacy classes under the action of the symmetry group $G$ including discrete transformations of (1.3). It turns out that only two classes of two-dimensional subalgebras exist for GW system (1.3). They can be represented by

$$
L_{2,1}=\left\{D_{1}, T_{1}+a H\right\}, \quad L_{2,2}=\left\{D_{2}, T_{2}+a H\right\}, \quad a \in \mathbb{C} .
$$

The corresponding invariant solutions of the reduced equations of GW system (1.3) lead to trivial constant solutions.

Let us now sum up the results. We remark that solutions of GW system obtained from one and two-dimensional reductions are elementary solutions, that is, constant, algebraic polynomial and exponential solutions. Thus the classical symmetry reduction method in the version presented here does not prove to be a very useful tool, since it leads to rather limited classes of solutions. In the framework of group theoretical methods applied to PDEs, there exists in the recent liturature several approaches such as nonclassical and conditional symmetry methods $[\mathbf{2 4 , 2 5 ]}$ which evolved in the process of extending Lie's classical theory of symmetry for PDEs. This approach consists basically in modifying the original system by adding to it certain differential constraints for which a symmetry criterion is identically satisfied. The overdetermined system of equations obtained in this way admits, in some cases, a larger class of Lie point symmetry groups and consequently, can provide other classes of solutions of the original system than the ones obtained by the classic approach. Thus, one of our objectives is to study the conditional symmetries of the GW system and look for new classes of solutions, which is presented in Section 4.

\section{THE SECOND ORDER SYSTEM.}

In our investigation it is more convenient to introduce a new dependent variable which links the GW system with the Euclidean sigma model. Such a link has been discovered first by Kenmotsu [26] for the linear Weierstrass equations inducing mean curvature surfaces. This will be extended to the case of the GW system, and it will allow us to establish several useful transformations in order to simplify the structure of system (1.3).

Let us define a new complex variable

$$
\rho=\frac{\psi_{1}}{\bar{\psi}_{2}}
$$

and its complex conjugate. Differentiating (3.1) with respect to $\partial$ and using the relation

$$
p=\left|\psi_{2}\right|^{2}\left(1+|\rho|^{2}\right),
$$

we obtain

$$
\partial \rho=\left(1+|\rho|^{2}\right)^{2} \psi_{2}^{2} .
$$


Note that $\partial \rho$ and $\psi_{2}^{2}$ have the same polar angle in the complex plane, since they are related by a real quantity $\left(1+|\rho|^{2}\right)^{2}$. Taking into account (3.1) we get the following transformation from the variable $\rho$ into the variables $\psi_{1}$ and $\psi_{2}$

$$
\psi_{1}=\epsilon \rho \frac{(\bar{\partial} \bar{\rho})^{1 / 2}}{1+|\rho|^{2}}, \quad \psi_{2}=\epsilon \frac{(\partial \rho)^{1 / 2}}{1+|\rho|^{2}}, \quad \epsilon= \pm 1 .
$$

Now, if $\psi_{1}$ and $\psi_{2}$ are solutions of GW system (1.3), then the function $\rho$ defined by (3.1) is a solution to the two-dimensional Euclidean sigma-model equations

$$
\text { (i) } \quad \partial \bar{\partial} \rho-\frac{2 \bar{\rho}}{1+|\rho|^{2}} \partial \rho \bar{\partial} \rho=0, \quad \text { (ii) } \quad \partial \bar{\partial} \bar{\rho}-\frac{2 \rho}{1+|\rho|^{2}} \partial \bar{\rho} \bar{\partial} \bar{\rho}=0 .
$$

Conversely, if $\rho$ is a solution of the sigma model (3.4), then the solutions $\psi_{1}$ and $\psi_{2}$ of GW system (1.3) have the form (3.3). Hence, some classes of solutions to GW system (1.3) can be obtained directly by applying the transformation (3.3) to the solutions of the sigma-model (3.4). For example, a very large class of solutions can be found simply by requiring the holomorphicity or antiholomorphicity of the function $\rho$. This choice for the function $\rho$ leads to so called splitting solutions (1.3), which satisfy the Laplace equation

$$
\partial \bar{\partial} \rho=0
$$

and have been extensively investigated in $[\mathbf{1 3}, \mathbf{1 5}]$. We discuss in detail a technique for constructing nonsplitting solutions of (1.3) for which $\partial \bar{\partial} \rho \neq 0$ holds in Sections 5,6 and 7. Note that the only trivial solution of GW system (1.3) is the null solution $\psi_{i} \equiv 0$, which corresponds to $\rho$ equal to any constant.

It is worth noting that WE system (1.3) possesses several conservation laws [6,27]. Among them, the conservation of current (1.7) and conservation of a potential function implied by

$$
\partial\left(\psi_{1}\right)^{2}+\bar{\partial}\left(\psi_{2}\right)^{2}=0
$$

and its complex conjugate,

$$
\bar{\partial}\left(\bar{\psi}_{1}\right)^{2}+\partial\left(\bar{\psi}_{2}\right)^{2}=0
$$

This means there exists a complex function $g: \mathbb{C} \rightarrow \mathbb{C}$ such that

$$
\psi_{1}=\epsilon(\bar{\partial} g)^{1 / 2}, \quad \psi_{2}=\epsilon(\partial g)^{1 / 2}, \quad \epsilon= \pm 1 .
$$

Substituting these into the WE system (1.3), we obtain the following second order system of PDEs

$$
\begin{gathered}
\partial \bar{\partial} g=2 i \epsilon\left[(\bar{\partial} g)(\partial \bar{g})^{1 / 2}(\partial g)^{1 / 2}+(\bar{\partial} g)^{1 / 2}(\partial g)(\bar{\partial} \bar{g})^{1 / 2}\right] \\
\bar{\partial} \partial \bar{g}=-2 i \epsilon\left[(\partial \bar{g})(\bar{\partial} g)^{1 / 2}(\bar{\partial} \bar{g})^{1 / 2}+(\partial \bar{g})^{1 / 2}(\bar{\partial} \bar{g})(\partial g)^{1 / 2}\right] .
\end{gathered}
$$

Hence, if $g$ is a solution of the system (3.8), then the functions $\psi_{1}$ and $\psi_{2}$ defined by (3.7) satisfies the GW system (1.3). Under the above conservation law, we can formulate the following

PROPOSITION 1. If $\psi_{1}$ and $\psi_{2}$ are solutions of WE system (1.3), then the current $J$ in terms of the function $\rho$ takes the form

$$
J(z, \bar{z})=-\frac{\partial \rho \partial \bar{\rho}}{\left(1+|\rho|^{2}\right)^{2}}
$$

and is a holomorphic function, $\bar{\partial} J=0$. 
PROOF. Indeed, substituting equations (3.3) into the expression (3.5)

$$
\begin{gathered}
J=\frac{\bar{\rho} \partial^{2} \rho}{2\left(1+|\rho|^{2}\right)^{2}}-\frac{\bar{\rho} \partial \rho}{\left(1+|\rho|^{2}\right)^{3}}(\rho \partial \bar{\rho}+\bar{\rho} \partial \rho)-\frac{\partial \rho \partial \bar{\rho}}{\left(1+|\rho|^{2}\right)^{3}}-\frac{\bar{\rho} \partial^{2} \rho}{2\left(1+|\rho|^{2}\right)^{2}}+\bar{\rho}^{2} \frac{(\partial \rho)^{2}}{\left(1+|\rho|^{2}\right)^{3}} \\
=-\frac{\partial \rho \partial \bar{\rho}}{\left(1+|\rho|^{2}\right)^{2}} .
\end{gathered}
$$

Differentiation of (3.10) yields

$$
\bar{\partial} J=\frac{(\bar{\partial} \partial \rho)(\partial \bar{\rho})+(\partial \rho)(\bar{\partial} \partial \bar{\rho})}{\left(1+|\rho|^{2}\right)^{2}}-2 \frac{(\partial \rho)(\partial \bar{\rho})(\rho \bar{\partial} \bar{\rho}+\bar{\rho} \bar{\partial} \rho)}{\left(1+|\rho|^{2}\right)^{3}} .
$$

Substituting equation (3.4) into (3.11), we obtain

$$
2 \bar{\rho}(\partial \rho)(\bar{\partial} \rho)(\partial \bar{\rho})+2 \rho(\partial \bar{\rho})(\bar{\partial} \bar{\rho})(\partial \rho)-2 \rho(\partial \rho)(\partial \bar{\rho})(\bar{\partial} \bar{\rho})-2 \bar{\rho}(\partial \rho)(\partial \bar{\rho})(\bar{\partial} \rho) \equiv 0 .
$$

Q.E.D.

PROPOSITION 2. If $\rho$ is a solution of sigma model system (3.4), then the conservation of potential (3.5) is identically satisfied.

PROOF. Indeed, substituting (3.3) into expression (3.5), we obtain

$$
\begin{gathered}
2 \rho \partial \rho \frac{\bar{\partial} \bar{\rho}}{\left(1+|\rho|^{2}\right)^{2}}+\rho^{2} \frac{\partial \bar{\partial} \bar{\rho}}{\left(1+|\rho|^{2}\right)^{2}}-2 \rho^{2} \frac{\bar{\partial} \bar{\rho}}{\left(1+|\rho|^{2}\right)^{3}}(\bar{\rho} \partial \rho+\rho \partial \bar{\rho}) \\
+\frac{\bar{\partial} \partial \rho}{\left(1+|\rho|^{2}\right)^{2}}-2 \frac{\partial \rho}{\left(1+|\rho|^{2}\right)^{3}}(\bar{\rho} \bar{\partial} \rho+\rho \bar{\partial} \bar{\rho})=0 .
\end{gathered}
$$

By virtue of sigma model equations (3.4), the equation above is an identity,

$$
\rho \partial \rho \bar{\partial} \bar{\rho}-\frac{\rho}{1+|\rho|^{2}}\left(1+|\rho|^{2}\right) \partial \rho \bar{\partial} \bar{\rho} \equiv 0
$$

Q.E.D.

\section{GROUP INVARIANT SOLUTIONS}

This Section is devoted to finding explicit solutions of GW system (1.3) based on transformation (3.3) and uses a variety of classes of invariant solutions of the sigma model (3.4). In order to construct and investigate solutions of sigma model (3.4) obtained by means of the symmetry reduction method $[\mathbf{1 7}, \mathbf{1 8}, \mathbf{2 4}]$ to ODEs, we need to find its symmetry group $G$, and then classify all subgroups $G_{i}$ of $G$ having generic orbits of codimension one in the space of independent variables. We then find the associated invariants of each of its subgroups $G_{i}$, and perform for each of these invariants the symmetry reduction of (3.4) to a system of ODEs and then solve these ODEs. The last step requires that a singularity analysis be carried out to determine whether they are of the Painlevé type, thus whether all their critical points are fixed, or independent of the initial data.

The reductions of sigma model (3.4) to systems of ODEs require considerations of one dimensional subalgebras of the symmetry algebra of (3.4). Using the MACSYMA program [19], we find that the classical symmetry groups $G$ of the sigma model equations (3.4) are the conformal and scaling transformations. The corresponding symmetry algebra $L$ is spanned by the vector fields

$$
X^{1}=\xi(z) \partial, \quad X^{2}=\eta(\bar{z}) \bar{\partial}, \quad D=\rho \partial_{\rho}-\bar{\rho} \partial_{\bar{\rho}} .
$$

Here, $\xi$ and $\eta$ are arbitrary functions of $z$ and $\bar{z}$, respectively. Now, since the distribution of the vector fields (4.1) is abelian, it determines that the algebra $\mathcal{L}$ can be decomposed as a direct sum of 
two infinite-dimensional simple Lie subalgebras with direct sum given by a one-dimensional algebra generated by $D$,

$$
\mathcal{L}=\left\{X_{1}\right\} \oplus\left\{X_{2}\right\} \oplus D
$$

If we assume that the functions $\xi$ and $\eta$ are analytic in a proper open subset $\Omega$ of $\mathbb{C}$, then they can be developed in a Laurent series. In this case, we can provide a basis for two centreless Virasoro algebras. Finite-dimensional subalgebras of $\left\{X_{1}\right\}$ and $\left\{X_{2}\right\}$ are spanned only by

$$
\{\partial\},\{\partial, z \partial\},\left\{\partial, z \partial, z^{2} \partial\right\}, \quad \cdots \quad \text { and } \quad\{\bar{\partial}\},\{\bar{z} \bar{\partial}\},\left\{\bar{\partial}, \bar{z} \bar{\partial}, \bar{z}^{2} \partial\right\}, \cdots
$$

respectively. The invariant solutions of the one-dimensional subalgebras (4.3) are only holomorphic or antiholomorphic functions and lead to splitting solutions of (3.4), which have been discussed in detail in [28]. According to $[\mathbf{1 7}, \mathbf{1 8 , 2 2}]$, we can apply the method of classifying finite subalgebras of direct-sum algebras (4.2). Here, we state only the result of this classification. We give below a list of representatives of conjugacy classes of one-dimensional subalgebras of symmetry algebra $\mathcal{L}$. The statement is that any one-dimensional subalgebra of $\mathcal{L}$ is conjugate under $G$ to give precisely one algebra $L_{1, j}(j=1, \cdots, 6)$ in the list and no two members of this list are mutually conjugate. The results are summarized by the following cases:

$$
\begin{array}{ccc}
L_{1,1}=\partial, & L_{1,2}=\bar{\partial}, & L_{1,3}=D \\
L_{1,4}=\partial+\bar{\partial}, & L_{1,5}=D+\partial, & L_{1,6}=D+\partial+\bar{\partial} .
\end{array}
$$

Note that complex conjugate subalgebras to these given in (4.4) were excluded in our classification list. The discrete subgroups of system (3.4) presented in Appendix A1 were used to restrict the range of parameters appearing in this classification.

For computational purposes, it is useful to examine the real system of PDEs equivalent to the two-dimensional Euclidean sigma model equations (3.4). If we introduce the polar coordinates

$$
\rho=R e^{i \phi}
$$

into system (3.4), then the real and imaginary parts of the so obtained equations have the following form for the unknown functions $R$ and $\phi$

$$
\begin{gathered}
\phi_{x x}+\phi_{y y}+\frac{2\left(1-R^{2}\right)}{R\left(1+R^{2}\right)}\left(R_{x} \phi_{x}+R_{y} \phi_{y}\right)=0 \\
R_{x x}+R_{y y}-\frac{R\left(1-R^{2}\right)}{1+R^{2}}\left(\phi_{x}^{2}+\phi_{y}^{2}\right)-\frac{2 R}{1+R^{2}}\left(R_{x}^{2}+R_{y}^{2}\right)=0 .
\end{gathered}
$$

Note that if we put $R=1$, then (4.6ii) is identically satisfied and the first one reduces to the Laplace equation for the phase $\phi$. This implies that $\phi$ has to be a periodic, harmonic function with a period equal to $2 \pi$. Otherwise, if the period of $\phi$ is not $2 \pi$, then the solution (4.6) may become a multivalued function. In our analysis, if we construct solutions that disobey the above restriction, then these solutions have to be excluded from our considerations in general.

Equations (4.6) are invariant under the discrete transformations generated by reflections (see Table 3) and also the inversion given by

$$
R \rightarrow \frac{1}{\eta}, \quad \phi \rightarrow \phi
$$

Making use of the MACSYMA program [19] for determining the symmetry algebra of a differential equation, we find that the symmetry algebra $\mathcal{L}$ of equations (4.6) is spanned by the following vector fields

$$
X^{1}=\frac{1}{2} \xi(z)\left(\partial_{x}-i \partial_{y}\right), \quad X^{2}=\frac{1}{2} \bar{\xi}(\bar{z})\left(\partial_{x}+i \partial_{y}\right), \quad \Phi=\partial_{\phi}
$$


where $\xi$ is an arbitrary function of $z=x+i y$ and $\bar{\xi}$ denotes its complex conjugate. Note the vector fields $X^{1}$ and $X^{2}$ can be rectified by any conformal transformation $z=z(u)$ and $\bar{z}=\bar{z}(\bar{u})$

$$
X^{1}=\xi(z) \partial \rightarrow \partial_{u}, \quad X^{2}=\bar{\xi}(\bar{z}) \bar{\partial} \rightarrow \partial_{\bar{u}}
$$

It is easy to check that this transformation preserves the form of the sigma model equations (3.4).

For computational purposes, it is useful to change the base of vector fields (4.8). The corresponding symmetry algebra $\mathcal{L}$ is spanned by

$$
\alpha^{ \pm}=X^{1} \pm \bar{X}^{1}=\frac{1}{2}\left\{(\xi \pm \bar{\xi}) \partial_{x}-i(\xi \mp \bar{\xi}) \partial_{y}\right\}, \quad \Phi=\partial_{\phi}
$$

since we have

$$
\alpha^{+}=X^{1}+\bar{X}^{1}=X^{2}+\bar{X}^{2}, \quad \alpha^{-}=-i\left(X^{1}-\bar{X}^{1}\right)=i\left(X^{2}-\bar{X}^{2}\right) .
$$

The algebra $\mathcal{L}$ can be decomposed as a direct sum of two infinite-dimensional simple Lie subalgebras with direct sum of a one-dimensional algebra generated by $\Phi$

$$
\mathcal{L}=\left\{\alpha^{+}\right\} \oplus\left\{\alpha^{-}\right\} \oplus\{\Phi\}
$$

Note that the vector field $\Phi$ commutes with the vector fields $\alpha^{+}$and $\alpha^{-}$, so it represents the center of the algebra $\mathcal{L}$. Assuming that the function $\xi$ is analytic in a proper open subset of $\mathcal{D} \subset \mathbb{C}$, we can develope $\xi$ as a power series and can provide the following basis

$$
\begin{gathered}
\alpha_{n}^{+}=X^{1}+\bar{X}^{1}=\frac{1}{2}\left\{(x+i y)^{n}+(x-i y)^{n}\right\} \partial_{x}-\frac{i}{2}\left\{(x+i y)^{n}-(x-i y)^{n}\right\} \partial_{y}, \\
\alpha_{n}^{-}=-i\left(X^{1}-\bar{X}^{1}\right)=-\frac{i}{2}\left\{(x+i y)^{n}-(x-i y)^{n}\right\} \partial_{x}-\frac{1}{2}\left\{(x+i y)^{n}+(x-i y)^{n}\right\} \partial_{y},
\end{gathered}
$$

where $n \in \mathbb{Z}^{+}$. The vector fields (4.10) generate an infinite-dimensional simple algebra that contains only one finite-dimensional subalgebra spanned by

$$
\begin{array}{cc}
P_{1}=\partial_{x} & P_{2}=\partial_{y} \\
D=x \partial_{x}+y \partial_{y} & L_{3}=y \partial_{x}-x \partial_{y} \\
C_{1}=\left(x^{2}-y^{2}\right) \partial_{x}+2 x y \partial_{y} & C_{2}=2 x y \partial_{x}-\left(x^{2}-y^{2}\right) \partial_{y}
\end{array}
$$

which corresponds to the case in which the index $n=0,1,2$ in equations (4.10). The physical interpretation of this Lie algebra is the following. The operators $P_{1}$ and $P_{2}$ generate translations in the $x$ and $y$ directions, respectively. The vector fields $D$ and $L_{3}$ correspond to dilation and rotation, respectively. The operators $C_{1}$ and $C_{2}$ generate two different types of conformal transformations. The nonvanishing commutation relations for the algebra (4.11) are given by

$$
\begin{array}{cccc}
{\left[C_{1}, L_{3}\right]=C_{1}} & {\left[C_{1}, D\right]=-C_{2} \quad\left[C_{1}, P_{1}\right]=-2 D \quad\left[C_{1}, P_{2}\right]=2 L_{3}} \\
{\left[C_{2}, L_{3}\right]=-C_{2} \quad\left[C_{2}, D\right]=C_{1} \quad\left[C_{2}, P_{1}\right]=-2 L_{3} \quad\left[C_{2}, P_{2}\right]=-2 D} \\
{\left[L_{3}, P_{1}\right]=P_{2} \quad\left[L_{3}, P_{2}\right]=-P_{1} \quad\left[D, P_{1}\right]=-P_{1} \quad\left[D, P_{2}\right]=-P_{2} .}
\end{array}
$$

These relations show that this algebra is isomorphic to the $O(3,1)$ algebra. We refer the reader to $[\mathbf{1 7}, \mathbf{2 9}]$ for details of the classification of subalgebras of the symmetry algebra $O(3,1)$ into conjugacy 
classes under the action of the symmetry group $G$. In our case, among all nonconjugate subalgebras, the ones that have generic orbits of codimension one in the space of independent variables and three in the space of independent and dependent variables $\{x, y, R, \phi\}$ reduce the original system (4.6) to a system of ODEs via the symmetry reduction method. The one-dimensional subalgebras are given by

$$
\begin{gathered}
P_{1}+b \Phi, \\
L_{3}+b \Phi, \\
D+b \Phi, \\
D+a L_{3}+b \Phi,
\end{gathered}
$$

where $a$ and $b$ are real parameters. The discrete subgroups (4.7) and (4.8) were used to restrict the range of parameters occuring in this classification. In order to find the reduction associated with subalgebras (4.13), we compute for each of them the corresponding invariants by solving the PDE

$$
X H(x, y, R, \phi)=0
$$

where $H$ is an auxilliary function of four variables $(x, y, R, \phi)$, and $X$ is one of the generators listed in (4.13). The solution of (4.14) is found by integrating the associated characteristic system. The result of it is that we find three invariants $\xi, R$ and $F$ which are listed for all cases in Table 4 . The orbits of the subgroups of $G$ for all considered cases (4.13) can be expressed in terms of two functions $R$ and $\phi$ in the following form

$$
R=R(\xi), \quad \phi=\alpha(x, y)+F(\xi), \quad \xi=\xi(x, y)
$$

where $\alpha$ and $\xi$ are given functions of $x$ and $y$ for each subalgebra. Here, the function $\xi$ is the symmetry variable of the invariance subgroup having generic orbits of codimension one. Substituting each specific form (4.15) into system (4.6) leads to the coupled system of ODEs in terms of the symmetry variable $\xi$ only

$$
\begin{gathered}
\text { (i) } \quad \ddot{R}-\frac{2 R}{1+R^{2}} \dot{R}^{2}-\frac{R\left(1-R^{2}\right)}{1+R^{2}} \dot{\phi}^{2}-\frac{\dot{g}}{g} \dot{R}-2 h \frac{R\left(1-R^{2}\right)}{1+R^{2}} \dot{\phi}-l \frac{R\left(1-R^{2}\right)}{1+R^{2}}=0, \\
\text { (ii) } \ddot{\phi}+2 \dot{R} \dot{\phi} \frac{\left(1-R^{2}\right)}{R\left(1+R^{2}\right)}-\frac{\dot{g}}{g} \dot{\phi}+2 h \frac{\left(1-R^{2}\right)}{R\left(1+R^{2}\right)} \dot{R}+s=0,
\end{gathered}
$$

where functions $g, h, l$ and $s$ are given for each of the subalgebras in Table 4 . The dot means differentiation with respect to $\xi$. The results of these calculations are summarized in Table 4 for each of the subalgebras.

The obtained reduced system of ODEs (4.16) has a form similar to the one obtained from the symmetry reduction method for the $C P^{1}$ model in $(2+1)$ dimensions as given in [30]. So, following the procedure presented in [30], equations (4.16) can be solved in an analogous way. The results of the Painlevé analysis for system (4.16) and the integration of these reduced ODEs in terms of elementary functions, Jacobi elliptic functions, or Painlevé transcendents are summarized in Table 4. The system of ODEs (4.16) for $R$ and $\phi$ can be decoupled. If we perform the transformation,

$$
\dot{\phi}=V-h,
$$


on equation (4.16ii), then the function $V$ has to satisfy the nonhomogeneous ODE

$$
\dot{V}+2 \dot{R} \frac{1-R^{2}}{R\left(1+R^{2}\right)} V-\frac{\dot{g}}{g} V-m=0
$$

where

$$
m=\dot{h}-\frac{\dot{g}}{g} h-s_{0}
$$

Let us consider separately two cases:

1 ) when $m=0$ equation (4.18) is an homogeneous ODE for the function $V$. This case corresponds to subalgebras $\left\{P_{1}+b \Phi_{1}\right\},\left\{L_{3}+b \Phi\right\}$ and $\left\{D+a L_{3}+b \Phi\right\}$ (see Table 4 ). The general integral of the homogeneous ODE (4.18) has the form

$$
V=A g \frac{\left(1+R^{2}\right)^{2}}{R^{2}}, \quad A \in \mathbb{R}
$$

Hence, transformation (4.17) becomes

$$
\dot{\phi}=A g \frac{\left(1+R^{2}\right)^{2}}{R^{2}}-h
$$

Elimination of $\dot{\phi}$ from (4.21) and (4.16i) gives

$$
\ddot{R}-\frac{2 R}{1+R^{2}} \dot{R}^{2}-\frac{\dot{g}}{g} \dot{R}-A^{2} g^{2} \frac{\left(1-R^{2}\right)\left(1+R^{2}\right)^{3}}{R^{3}}+\left(h^{2}-l\right) \frac{R\left(1-R^{2}\right)}{1+R^{2}}=0 .
$$

2) when $m \neq 0$ equation (4.19) is a nonhomogeneous ODE for the function $V$. This case is related to subalgebra $D+b \Phi$, as in Table 4 .

The general solution of ODE (4.18) is obtained by the method of variation of parameters and has the following form

$$
V=A(\xi) g \frac{\left(1+R^{2}\right)^{2}}{R^{2}}
$$

with

$$
A(\xi)=\int \frac{m R^{2}}{g\left(1+R^{2}\right)^{2}} d \xi^{\prime}
$$

Substituting (4.23) and (4.24) into (4.17), we can eliminate $\dot{\phi}$ from (4.16i), and we get (4.22). In order to construct solutions of (4.22), we have to analyze the singularity structure in the sense of Painlevé and Gambier [31,32] and transform these equations to one of the standard forms listed in [33]. If we perform the transformation of the dependent variable in (4.22)

$$
R(\xi)=(-U(\xi))^{1 / 2},
$$

then the function $U$ has to satisfy the second order ODE

$$
\ddot{U}=\left(\frac{1}{2 U}-\frac{1}{1-U}\right) \dot{U}^{2}-\frac{\dot{g}}{g} \dot{U}+\frac{2 C^{2}}{g^{2}} \frac{(1+U)(1-U)^{3}}{U} .
$$

Changing the independent variable

$$
\eta=\int_{\xi_{0}}^{\xi} \frac{d t}{g(t)}, \quad g(t) \neq 0
$$


in (4.26), we obtain the following ODE in the $\eta$ variable

$$
\ddot{U}=\left(\frac{1}{2 U}-\frac{1}{1-U}\right) \dot{U}^{2}+\frac{2 C^{2}}{U}(1+U)(1-U)^{3} .
$$

If $C=0$, then from (4.14), $\phi=\phi_{0}$, and equation (4.20) becomes Eq. PXXXVII listed by Ince [33] with the solution given by

$$
U=\tanh ^{2}\left(K_{1} \eta+K_{2}\right), \quad K_{1}, K_{2} \in \mathbb{R}
$$

If $C \neq 0$, then equation (4.20) is equation PXXXVIII listed in Ince [33], where in Ince's notation, $\beta=-\alpha=2 C^{2}$ and $\gamma=\delta=0$. This equation admits a first integral and can be reduced to a first-order ODE for $U$

$$
U^{\prime}=-4 C^{2} U^{4}+4 K U^{3}+8\left(C^{2}-K\right) U^{2}+4 K U-4 C^{2}, \quad K \in \mathbb{C},
$$

where differentiation is with respect to $\eta$. Equation (4.30) can be written in equivalent form for $C \neq 0$

$$
U^{\prime 2}=-4 C^{2}\left(U-U_{1}\right)\left(U-U_{2}\right)\left(U-U_{3}\right)\left(U-U_{4}\right),
$$

where $U_{i}, i=1, \cdots, 4$ denote the constant roots of the right-hand side of (4.31). These roots can be expressed in terms of the constants $C$ and $K$. It is well known [30] that behavior of the solution of (4.31) depends upon the relationships between the roots of a quartic polynomial of the form of the right hand side of (4.31). This equation is a known equation which can be solved in terms of elliptic Jacobi functions (see Byrd and Friedman [34]) or in the degenerate cases, as when the roots have multiplicity greater than one, in terms of elementary algebraic functions with one or two simple poles, trigonometric and hyperbolic solitons.

Localized solutions can also be obtained. Suppose that $C=0$, then (4.31) takes the form

$$
\dot{U}^{2}=\left(4 K U^{2}-2(B+4 K) U+4 K\right) U
$$

When $K=0$, this can be integrated easily to give

$$
U=D e^{ \pm \sqrt{-2 B} \eta}
$$

When $K \neq 0$, one can write the quadratic in factorized form

$$
\dot{U}^{2}=4 K\left(U-U_{1}\right)\left(U-U_{2}\right) U
$$

where the roots are given as follows,

$$
U_{1,2}=(l+1) \pm \sqrt{l(l+2)}
$$

with $l=B / 4 K$. We have summarized all individual possibilities in Tables 5, 6 and 7 .

As an example to illustrate how this method works, let us write the functions $\psi_{i}$ for a particular case of roots and two of the symmetries which appear in Table 4. Suppose that three of the roots are the same, so that $U_{4}=U_{3}=U_{2}<U \leq U_{1}<0$. Equation (4.31), can be intergated and we can solve to obtain $U$ as given in Table 7 as follows

$$
U=\frac{U_{2} C^{2}\left(U_{1}-U_{2}\right)^{2}\left(\eta-\eta_{0}\right)^{2}+U_{1}}{C^{2}\left(U_{1}-U_{2}\right)^{2}\left(\eta-\eta_{0}\right)^{2}+1}
$$


For the symmetries $\left\{P_{1}+b \Phi\right\}$ and $\left\{D+a L_{3}+b \Phi\right\}$ in Table $4, g=g_{0}$ is constant, so from (4.27), we obtain

$$
\eta-\eta_{0}=\frac{1}{g_{0}}\left(\xi-\xi_{0}\right)
$$

and putting $R=\sqrt{-U}$ in $(4.20)$,

$$
F=-g_{0} A \int \frac{(1-U)^{2}}{U} d \xi-h \xi
$$

For this case and symmetry $\left\{P_{1}+b \Phi\right\}$, for which the symmetry variable is given by $\xi=y, h=0$, and $\phi=b x+F(\xi)$. Then $\rho$ can be written in the following form

$$
\begin{aligned}
\rho=R e^{i \phi} & =\sqrt{-\frac{U_{2} C^{2} g_{0}^{-2}\left(U_{1}-U_{2}\right)^{2}\left(y-y_{0}\right)^{2}+U_{1}}{C^{2} g_{0}^{-2}\left(U_{1}-U_{2}\right)^{2}\left(y-y_{0}\right)^{2}+1}} \exp \left[i \left(b x+\frac{g_{0}^{2} A}{C} \tan ^{-1}\left(\left(y-y_{0}\right)\left(U_{2}-U_{1}\right) g_{0}^{-1} C\right)\right.\right. \\
& \left.\left.-\frac{g_{0}^{2} A\left(U_{2}-U_{1}\right)}{C U_{2}\left(U_{1} U_{2}\left(U_{1}-U_{2}\right)^{2}\right)^{1 / 2}} \tan ^{-1}\left(\frac{U_{2} C\left(U_{2}-U_{1}\right)^{2}\left(y-y_{0}\right)}{g_{0}\left(U_{1} U_{2}\left(U_{1}-U_{2}\right)^{2}\right)^{1 / 2}}\right)-g_{0} A \frac{\left(U_{2}-1\right)^{2}}{U_{2}} y\right)\right] .
\end{aligned}
$$

To evaluate the $\psi_{i}$, one next calculates the derivatives $\partial \rho$ and $\bar{\partial} \bar{\rho}$, and then substitutes these into (3.3). To abbreviate the notation, let's define the quantities $K=C^{2} g_{0}^{-2}\left(U_{1}-U_{2}\right)^{2}, f=$ $g_{0}\left(U_{1} U_{2}\left(U_{1}-U_{2}\right)^{2}\right)^{1 / 2}$ and $Q=g_{0}^{2}\left(A\left(U_{2}-U_{1}\right) / C U_{2}\right)$. Then for the case of the first symmetry, the $\psi_{i}$ are as follows

$$
\begin{aligned}
& \psi_{1}=\epsilon \frac{\rho}{1-U}[-\frac{i U_{2} K\left(\xi-\xi_{0}\right)}{2 R K\left(\xi-\xi_{0}\right)^{2}+1}-\frac{1}{2} i R K\left(\xi-\xi_{0}\right)-i R\left(\frac{1}{2} b+\frac{i g_{0} A\left(U_{2}-U_{1}\right)}{2\left(1+\left(U_{2}-U_{1}\right)^{2} g_{0}^{-2} C^{2}\left(\xi-\xi_{0}\right)^{2}\right)}\right. \\
&\left.\left.-\frac{i g_{0} Q U_{2} C\left(U_{2}-U_{1}\right)^{2}}{2\left(f^{2}+U_{2}^{2} C^{2}\left(U_{2}-U_{1}\right)^{4}\left(\xi-\xi_{0}\right)^{2}\right)}-\frac{i g_{0} A\left(U_{2}-1\right)^{2}}{2 U_{2}}\right)\right]^{1 / 2} \exp (-i \phi / 2), \\
& \psi_{2}=\frac{\epsilon}{1-U}\left[\frac{i U_{2} K\left(\xi-\xi_{0}\right)}{2 R K\left(\xi-\xi_{0}\right)^{2}+1}+\frac{1}{2} i R K\left(\xi-\xi_{0}\right)+i R\left(\frac{1}{2} b-\frac{i g_{0} A\left(U_{2}-U_{1}\right)}{2\left(1+\left(U_{2}-U_{1}\right)^{2} g_{0}^{-2} C^{2}\left(\xi-\xi_{0}\right)^{2}\right)}\right.\right. \\
&\left.\left.+\frac{i g_{0} Q C U_{2}\left(U_{2}-U_{1}\right)^{2}}{2\left(f^{2}+U_{2}^{2} C^{2}\left(U_{2}-U_{1}\right)^{4}\left(\xi-\xi_{0}\right)^{2}\right)}+\frac{i g_{0} A\left(U_{2}-1\right)^{2}}{2 U_{2}}\right)\right]^{1 / 2} \exp (i \phi / 2) .
\end{aligned}
$$

For symmetry $\left\{D+a L_{3}+b \Phi\right\}$, the function $\rho$ can be written down in terms of the symmetry variable abbreviated as $\xi=\ln \sqrt{x^{2}+y^{2}}+(1 / a) \tan ^{-1} x / y$ and $h=2 b /\left(1+a^{2}\right)$

$$
\begin{gathered}
\rho=R e^{i \phi}=\sqrt{-\frac{U_{2} C^{2} g_{0}^{-2}\left(U_{1}-U_{2}\right)^{2}\left(\xi-\xi_{0}\right)^{2}+U_{1}}{C^{2} g_{0}^{-2}\left(U_{1}-U_{2}\right)^{2}\left(\xi-\xi_{0}\right)^{2}+1} \exp \left[i \left(-\frac{b}{a} \tan ^{-1} \frac{x}{y}+\frac{g_{0}^{2} A}{C} \tan ^{-1}\left(\left(\xi-\xi_{0}\right)\left(U_{2}-U_{1}\right) g_{0}^{-1} C\right)\right.\right.} \\
\left.-\frac{g_{0}^{2} A\left(U_{2}-U_{1}\right)}{C U_{2}\left(U_{1} U_{2}\left(U_{1}-U_{2}\right)^{2}\right)^{1 / 2}} \tan ^{-1}\left(\frac{U_{2} C\left(U_{2}-U_{1}\right)^{2}\left(\xi-\xi_{0}\right)}{\left.g_{0}\left(U_{1} U_{2}\left(U_{1}-U_{2}\right)^{2}\right)^{1 / 2}\right)}-g_{0} A \frac{\left(U_{2}-1\right)^{2}}{U_{2}} \xi+\frac{2 b}{1+a^{2}} \xi\right)\right] .
\end{gathered}
$$

Defining the additional variables

$$
s_{1}=1+\frac{x^{2}}{y^{2}}, \quad s_{2}=\frac{1}{2 i y}+\frac{x}{2 y^{2}}, \quad \tilde{s}_{2}=\frac{1}{2 i y}-\frac{x}{2 y^{2}}, \quad w_{1}=\frac{1}{2 z}+\frac{i s_{2}}{a s_{1}}, \quad w_{2}=\frac{1}{2 \bar{z}}+\frac{i \tilde{s}_{2}}{a s_{1}} .
$$

and with $K, f$ and $Q$ defined above, the functions $\psi_{i}$ are calculated by differentiating $\rho$ and using $(3.3)$

$$
\psi_{1}=\epsilon \frac{\rho}{1-U}\left[-\frac{U_{2} K\left(\xi-\xi_{0}\right) w_{2}}{\left(K\left(\xi-\xi_{0}\right)^{2}+1\right) R}+\frac{\left(U_{2} K\left(\xi-\xi_{0}\right)^{2}+U_{1}\right) K\left(\xi-\xi_{0}\right) w_{2}}{R\left(K\left(\xi-\xi_{0}\right)^{2}+1\right)^{2}}-i R\left(-\frac{i b \tilde{s}_{2}}{a s_{1}}\right.\right.
$$




$$
\begin{gathered}
\left.\left.+\frac{g_{0} A\left(U_{2}-U_{1}\right) w_{2}}{1+\left(U_{2}-U_{1}\right)^{2} g_{0}^{-2} C^{2}\left(\xi-\xi_{0}\right)^{2}}-\frac{g_{0} Q C U_{2}\left(U_{2}-U_{1}\right)^{2} w_{2}}{f^{2}+U_{2}^{2} C^{2}\left(U_{2}-U_{1}\right)^{4}\left(\xi-\xi_{0}\right)^{2}}-\frac{g_{0} A\left(U_{2}-1\right)^{2}}{U_{2}} w_{2}+\frac{2 b}{1+a^{2}} w_{2}\right)\right]^{1 / 2} \\
\cdot \exp (-i \phi / 2), \\
\psi_{2}=\frac{\epsilon}{1-U}\left[-\frac{U_{2} K\left(\xi-\xi_{0}\right) w_{1}}{\left(K\left(\xi-\xi_{0}\right)^{2}+1\right) R}+\frac{\left(U_{2} K\left(\xi-\xi_{0}\right)^{2}+U_{1}\right) K\left(\xi-\xi_{0}\right) w_{1}}{R\left(K\left(\xi-\xi_{0}\right)^{2}+1\right)^{2}}+i R\left(-\frac{i b s_{2}}{a s_{1}}\right.\right. \\
\left.\left.+\frac{g_{0} Q C U_{2}\left(U_{2}-U_{1}\right)^{2} w_{1}}{1+\left(U_{2}-U_{1}\right)^{2} g_{0}^{-2} C^{2}\left(\xi-\xi_{0}\right)^{2}}-\frac{g_{0} A\left(U_{2}-1\right)^{2}}{f^{2}+U_{2}^{2} C^{2}\left(U_{2}-U_{1}\right)^{4}\left(\xi-\xi_{0}\right)^{2}}-\frac{2 b}{1+a^{2}} w_{1}\right)\right]^{1 / 2} \\
\cdot \exp (i \phi / 2) .
\end{gathered}
$$

A class of solutions of (4.18) different from those obtained above can be constructed by introducing the change of independent variables

$$
\eta=\int g(\xi) d \xi
$$

into (4.17). We obtain

$$
U^{\prime \prime}=\left(\frac{1}{2 U}+\frac{1}{U-1}\right) U^{\prime 2}+2 \frac{g^{\prime}}{g} U^{\prime}-\frac{2 C^{2}}{g^{4}}(1-U)^{2}\left(\frac{1}{U}-U\right)
$$

where differentiation is with respect to $\eta$. Note that if $g=g_{0}$, then we obtain the previously discussed case PXXXVIII. The symmetry reduction to ODE (4.24) with $g=A \xi$ is related to the symmerty algebra $L_{3}$. In this case, equation (4.24) is the standard form for the fifth Painlevé transcendent [33], where in Ince's notation, $\beta=-\alpha=2 C^{2} / A^{4}$ and $\gamma=\delta=0$. However, if we make the transformation

$$
g=\exp \left(\int_{\xi_{0}}^{\xi} r(\eta) d \eta\right)
$$

for which the function $r$ is given by one of the following two cases

$$
r=\frac{1}{A \xi^{2}}, \quad r=\frac{2 \xi}{A\left(1+\xi^{2}\right)^{2}},
$$

which are related to the algebra $L_{3}$ or $D$, respectively, then equation (4.24) is Eq PXXXX listed in [33]. According to [33], equation (4.24) can be reduced to the first order ODE after the transformation

$$
\frac{1}{u-1} u^{\prime}-B e^{-2 \int r d \eta} u=-2 w, \quad B \in \mathbb{R}
$$

to a Riccati equation of the form

$$
w^{\prime}+w^{2}+2 r w-\frac{1}{2} B^{2} e^{-4 \int r d \eta}=E e^{-2 \int r d \eta}
$$

If $g$ in equation (4.17) is a constant, then we can perform a new transformation on the independent variable

$$
\eta=e^{i g_{0}\left(\xi-\xi_{0}\right)}
$$

into (4.17) and obtain

$$
U^{\prime \prime}=\left(\frac{1}{2 U}+\frac{1}{U-1}\right) U^{\prime 2}-\frac{1}{\eta} U^{\prime}+\frac{2 C^{2}}{g^{4}}(1-U)^{2}\left(\frac{1}{U}-U\right) .
$$


This is the equation for the fifth Painlevé transcendent, where in Ince's notation, $\beta=-\alpha=2 C^{2} / g_{0}^{4}$ and $\gamma=\delta=0$, which is related to the reduction obtained from the symmetry algebras $P_{1}$ and $\left\{D+a L_{3}\right\}$, respectively.

5 THE NON-SPLITTING SOLUTIONS OF THE GENERALIZED WEIERSTRASS SYSTEM

Now we discuss the case when some classes of solutions of GW system (1.3) can be obtained from transformation (3.3) for which the solutions of the sigma model (3.4) are invariant under the scaling transformation $D$ as given in (4.1). This means that we subject system (1.3) to the algebraic constraint

$$
|\rho|^{2}=1
$$

It follows from (5.1) that sigma model (3.4) can be written

$$
\partial \bar{\partial} \rho-\bar{\rho} \partial \rho \bar{\partial} \rho=0, \quad \bar{\partial} \partial \bar{\rho}-\rho \partial \bar{\rho} \bar{\partial} \bar{\rho}=0,
$$

and has a solution of the exponential form

$$
\rho=e^{i \varphi}
$$

where $\varphi$ is any real harmonic function of $z$ and $\bar{z}$, since from (3.4), we get the following identity

$$
\partial \bar{\partial} \rho-\bar{\rho} \partial \rho \bar{\partial} \rho=e^{i \varphi}(i \partial \bar{\partial} \varphi-\partial \varphi \bar{\partial} \varphi)+e^{i \varphi}\left(e^{-i \bar{\varphi}} e^{i \varphi} \partial \varphi \bar{\partial} \varphi\right) \equiv 0 .
$$

The transformation (3.3) becomes

$$
\psi_{1}=\frac{\epsilon}{2} e^{i \varphi / 2}(\bar{\partial} \varphi)^{1 / 2}, \quad \psi_{2}=\frac{\epsilon}{2} e^{i \varphi / 2}(\partial \varphi)^{1 / 2}, \quad \epsilon= \pm 1
$$

and satisfies GW system (1.3).

In this Section, we shall study nonsplitting solutions of overdetermined system (3.4) and (5.1) depending on one arbitrary complex valued function of one complex variable $z$ and its complex conjugate

$$
\rho=\frac{f(z)}{\bar{f}(\bar{z})} .
$$

In this case, transformation (3.3) defined in terms of a function $f$ takes the form

$$
\psi_{1}=\frac{\epsilon}{2 \bar{f}}(f \bar{\partial} \bar{f})^{1 / 2}, \quad \psi_{2}=\frac{\epsilon}{2 \bar{f}}(\bar{f} \partial f)^{1 / 2}, \quad p=\frac{1}{2}\left|\frac{\partial f}{f}\right| .
$$

The associated surface determined from equations (1.4) becomes

$$
\begin{gathered}
X_{1}+i X_{2}=\frac{i}{2} \int_{\gamma}\left(\frac{\bar{f} \partial f}{f^{2}} d z^{\prime}-\frac{\bar{\partial} \bar{f}}{\bar{f}} d \bar{z}^{\prime}\right), \\
X_{1}-i X_{2}=\frac{i}{2} \int_{\gamma}\left(\frac{\partial f}{f} d z^{\prime}-\frac{f \bar{\partial} \bar{f}}{\bar{f}^{2}} d \bar{z}^{\prime}\right), \\
X_{3}=-\frac{1}{2} \int_{\gamma}\left(\frac{\partial f}{f} d z^{\prime}+\frac{\bar{\partial} \bar{f}}{\bar{f}} d \bar{z}^{\prime}\right) .
\end{gathered}
$$

The induced metric on the surface and its Gaussian curvature (1.5) are given by

$$
d s^{2}=4 p^{2} d z d \bar{z}=\left|\frac{\partial f}{f}\right|^{2} d z d \bar{z}, \quad K=-p^{-2} \partial \bar{\partial}(\ln p)=0,
$$


respectively.

Note that if function $\rho$ is a solution of (3.4), and $\rho$ satisfies algebraic condition (5.1), then both of the functions $\rho^{-1}$ and $\bar{\rho}$ are also solutions of equation (3.4). In fact, the derivatives of $\rho$ are

$$
\partial \bar{\rho}=-\frac{1}{\rho^{2}} \partial \rho, \quad \bar{\partial} \bar{\rho}=-\frac{1}{\rho^{2}} \bar{\partial} \rho, \quad \partial \bar{\partial} \bar{\rho}=\frac{2}{\rho^{3}}(\partial \rho \bar{\partial} \rho)-\frac{1}{\rho^{2}} \partial \bar{\partial} \rho .
$$

Substituting (5.5) into equation (3.4), we obtain

$$
2 \frac{\partial \rho \bar{\partial} \rho}{\rho^{3}}-\frac{1}{\rho^{2}} \partial \bar{\partial} \rho-\frac{1}{\rho^{3}} \partial \rho \bar{\partial} \rho=0,
$$

which is just the identity

$$
\partial \bar{\partial} \rho-\frac{1}{\rho} \partial \rho \bar{\partial} \rho \equiv 0
$$

since $\bar{\rho}=1 / \rho$, and whenever (3.4) holds. Then we have the following the following Propositions.

PROPOSITION 3. Suppose that for any complex-valued function $F$ of class $C^{1}$, the function $\rho$ satisfies the algebraic condition (5.1) and the differential constraints

$$
\partial \rho=F(z) \rho, \quad \bar{\partial} \rho=-\bar{F}(\bar{z}) \rho .
$$

Then the complex valued function $\rho$ is a nonsplitting solution of the system (3.4). The associated surface has zero Gaussian curvature (1.5).

PROOF. Note that from equation (5.6), we have

$$
\bar{\partial} \partial \rho=F(z) \bar{\partial} \rho .
$$

Thus, system (3.4) is identically satisfied, since

$$
F(\bar{\partial} \rho)-\bar{\rho}(F \rho)(-F \rho)=F(-\bar{F} \rho)-F \bar{F} \rho \equiv 0
$$

holds. Moreover, from (3.3), we get

$$
\partial \psi_{1}=\frac{\epsilon}{4}(\bar{F} \rho)^{1 / 2} F, \quad \bar{\partial} \psi_{2}=-\frac{\epsilon}{4}(F \rho)^{1 / 2} \bar{F}, \quad p=\frac{1}{2}(F \bar{F})^{1 / 2}, \quad \epsilon= \pm 1 .
$$

So in view of (4.7), the GW system is also identically satisfied. Moreover, we have

$$
\partial \ln p=\frac{\partial p}{p}=\frac{\partial F}{2 F}
$$

which implies that $\bar{\partial}(\partial p)=0$, since $F$ is a function of only $z$. Thus from formula (1.5), the Gaussian curvature $K$ is zero. QED.

We discuss now the possibility of constructing more general classes of solutions of GW system (1.3) which are based on nonlinear superpositions of $N$ elementary solutions of sigma model (3.4).

PROPOSITION 4. (Factorization) Suppose that for each $i=1, \cdots, N$ the complex valued functions $\rho_{i}$ satisfy $\left|\rho_{i}\right|^{2}=1$ and system (3.4). Then the product function

$$
\rho=\prod_{i=1}^{N} \rho_{i}
$$


is also a solution of (3.4). The corresponding solution of the WE system takes the following factorized form

$$
\psi_{1}=\frac{1}{2} \epsilon \prod_{i=1}^{N} \rho_{i}\left(\bar{\partial} \prod_{j=1}^{N} \bar{\rho}_{j}\right)^{1 / 2}, \quad \psi_{2}=\frac{1}{2} \epsilon\left(\partial \prod_{j=1}^{N} \rho_{j}\right)^{1 / 2} .
$$

PROOF. It suffices to prove this for $N=2$, and then to invoke induction to extend this to any $N$. Suppose that $\rho_{1}, \rho_{2}$ are solutions to (3.4). Substituting the function $\rho=\rho_{1} \rho_{2}$ into (3.4), we obtain

$$
\begin{gathered}
\left(\bar{\partial} \partial \rho_{1}\right) \rho_{2}+\partial \rho_{1} \bar{\partial} \rho_{2}+\bar{\partial} \rho_{1} \partial \rho_{2}+\rho_{1} \bar{\partial} \partial \rho_{2}-\bar{\rho}_{1} \rho_{2} \partial \rho_{1} \bar{\partial} \rho_{1}-\bar{\partial} \rho_{1} \partial \rho_{2}-\partial \rho_{1} \bar{\partial} \rho_{2}-\rho_{1} \bar{\rho}_{2} \partial \rho_{2} \bar{\partial} \rho_{2} \\
=\rho_{2}\left(\bar{\partial} \partial \rho_{1}-\bar{\rho}_{1} \partial \rho_{1} \bar{\partial} \rho_{1}\right)+\rho_{1}\left(\bar{\partial} \partial \rho_{2}-\bar{\rho}_{2} \partial \rho_{2} \bar{\partial} \rho_{2}\right)=0 .
\end{gathered}
$$

Thus, the product of two solutions is a solution. Consequently, proceeding inductively from the product form of $\rho$ given in (5.7), if the equation is satisfied for a solution with $k$ factors, it is satisfied for a solution with $k+1$ factors. Substituting (5.7) into (3.3), we obtain $\psi_{1}$ and $\psi_{2}$ which satisfy system (1.1). QED.

Propositions 3 and 4 provide us with the possibility of constructing a class of nonsplitting solutions of equations (1.3). We now present several examples of such solutions.

1. Let us discuss now the construction of an algebraic multi-soliton solution of the GW system (1.3). First, we look for a particular class of rational solutions of (3.4) admitting simple poles at the points $\bar{z}=\bar{a}_{j}$,

$$
\rho_{j}=\frac{z-a_{j}}{\bar{z}-\bar{a}_{j}}, \quad a_{j} \in \mathbb{C}, \quad j=1, \cdots, N .
$$

By virtue of Propositions 3 and 4, a more general case than (5.8) can be constructed by taking products over $j$ in (5.8), when the solution $\rho_{j}$ of (3.4) admits an arbitrary number of simple poles,

$$
\rho=\prod_{k=1}^{N} \frac{z-a_{k}}{\bar{z}-\bar{a}_{k}}, \quad a_{k} \in \mathbb{C},
$$

where we assume that all $a_{k}$ are distinct. Note that the complex function $\rho$ admits a removable singularity since

$$
\lim _{z \rightarrow a_{k}}\left(\bar{z}-\bar{a}_{k}\right)\left(\frac{z-a_{k}}{\bar{z}-\bar{a}_{k}}\right)=0
$$

and thus

$$
\lim _{z \rightarrow a_{k}}\left(\bar{z}-\bar{a}_{k}\right) \prod_{j=1}^{N}\left(\frac{z-a_{j}}{\bar{z}-\bar{a}_{j}}\right)=0 .
$$

In that case the function $\rho$ satisfies both conditions $\partial \bar{\partial} \rho \neq 0$ and $|\rho|^{2}=1$. Substituting (5.9) into (3.4), we obtain that equations (3.4) are satisfied identically. The first derivatives of $\rho$ are given by

$$
\partial \rho=\sum_{j=1}^{N} \frac{1}{\left(z-a_{j}\right)} \rho \equiv F(z) \rho, \quad \bar{\partial} \rho=-\sum_{j=1}^{N} \frac{1}{\left(\bar{z}-\bar{a}_{j}\right)} \rho \equiv-\bar{F}(\bar{z}) \rho .
$$

Substituting (5.9) and (5.10) into (3.3), we determine the explicit form of an algebraic multi-soliton solution of the WE system

$$
\psi_{1}=\frac{\epsilon}{2}\left(\sum_{j=1}^{N} \frac{1}{\left(\bar{z}-\bar{a}_{j}\right)} \rho\right)^{1 / 2}, \quad \psi_{2}=\frac{\epsilon}{2}\left(\sum_{j=1}^{N} \frac{1}{\left(z-a_{j}\right)} \rho\right)^{1 / 2}, \quad \epsilon= \pm 1 .
$$


For the case $N=1$, substituting (5.11) into relations (1.4), we obtain that the corresponding constant mean curvature surface is determined by

$$
\left(X_{1}^{2}+X_{2}^{2}\right)^{2}-\left(2+\frac{a^{2}}{4} e^{2 X_{3}}\right)\left(X_{1}^{2}+X_{2}^{2}\right)+\frac{a^{2}}{2} e^{2 X_{3}} X_{2}+1-\frac{a^{2}}{4} e^{2 X_{3}}=0 .
$$

2. Another class of rational solutions can be written down by noting that, by Proposition 4 , a new solution can be found by taking products of $\rho_{k}$ with itself $k$ times and so

$$
\rho_{k}=\left(\frac{z-a_{k}}{\bar{z}-\bar{a}_{k}}\right)^{k}
$$

satisfies identically equation (3.4) for any integer $k$ and complex $a_{k}$. Consequently, we can generalize the form of this solution to another type of non-splitting multi-soliton solution of (3.4) by applying Proposition 4 again and forming products over $k$. This solution is generated by a $\rho$ of the form

$$
\rho=\prod_{k=1}^{N}\left(\frac{z-a_{k}}{\bar{z}-\bar{a}_{k}}\right)^{k}
$$

Note that for each $a_{k}$, the function $\rho$ has a removable singularity. So in this case, the function $\rho$ satisfies $|\rho|^{2}=1$, and the derivatives of $\rho$ are given by

$$
\partial \rho=\sum_{k=1}^{N} \frac{k}{z-a_{k}} \rho \equiv H \rho, \quad \bar{\partial} \rho=-\sum_{k=1}^{N} \frac{k}{\bar{z}-\bar{a}_{k}} \rho \equiv \bar{H} \rho .
$$

Substituting (5.12) and (5.13) into equations (3.4), we find that

$$
\bar{\partial} \partial \rho-\bar{\rho} \partial \rho \bar{\partial} \rho=-\sum_{k=1}^{N} \frac{k}{z-a_{k}} \sum_{j=1}^{N} \frac{j}{\bar{z}-\bar{a}_{j}} \rho+\bar{\rho} \sum_{k=1}^{N} \frac{k}{z-a_{k}} \rho \sum_{j=1}^{N} \frac{j}{\bar{z}-\bar{a}_{j}} \rho=0
$$

From (5.12) and (5.13), we can determine through the transformation (3.3) the explicit form of an algebraic multi-soliton solution of the WE system

$$
\psi_{1}=\frac{\epsilon}{2}\left(\sum_{k=1}^{N} \frac{k}{\left(\bar{z}-\bar{a}_{k}\right)} \rho\right)^{1 / 2}, \quad \psi_{2}=\frac{\epsilon}{2}\left(\sum_{k=1}^{N} \frac{k}{\left(z-a_{k}\right)} \rho\right)^{1 / 2} .
$$

Similarly, these solutions as in the previous case (5.11) admit also simple poles at $z=a_{k}$

3. An interesting class of non-splitting solution can be found by applying Proposition 4 to $\rho_{k}$ a total of $n$ times for a fixed number $n$, to obtain a new solution, and then applying Proposition 4 again by taking products over $k$

$$
\rho=\prod_{k=1}^{N}\left(\frac{z-a_{k}}{\bar{z}-\bar{a}_{k}}\right)^{n}
$$

The function $\rho$ satisfies (5.1), and the derivatives of $\rho$ are given by,

$$
\partial \rho=n \sum_{j=1}^{N} \frac{1}{\left(z-a_{j}\right)} \rho, \quad \bar{\partial} \rho=-n \sum_{j=1}^{N} \frac{1}{\left(\bar{z}-\bar{a}_{j}\right)} \rho .
$$


From (3.3), the solution of WE system (1.3) in this case takes the form

$$
\psi_{1}=\frac{\epsilon}{2}\left(n \sum_{j=1}^{N} \frac{1}{\left(\bar{z}-\bar{a}_{j}\right)} \rho\right)^{1 / 2}, \quad \psi_{2}=\frac{\epsilon}{2}\left(n \sum_{j=1}^{N} \frac{1}{\left(z-a_{j}\right)} \rho\right)^{1 / 2} .
$$

Note that the function $\psi_{i}$ admits only simple poles.

4. There exists an exponential class of non-splitting solution of GW system (1.3) which is generated by the products of functions of the form $\rho_{j}=e^{\bar{\alpha}_{i} z-\alpha_{i} \bar{z}}$ as follows

$$
\rho=\prod_{j=1}^{N} \rho_{j}=e^{\bar{A} z-A \bar{z}}
$$

This satisfies condition (5.1), and generates, using Proposition 4, the following class of solutions of GW system (1.3),

$$
\psi_{1}=\frac{\epsilon}{2} A^{1 / 2} e^{(\bar{A} z-A \bar{z}) / 2}, \quad \psi_{2}=\frac{\epsilon}{2} \bar{A}^{1 / 2} e^{(\bar{A} z-A \bar{z}) / 2} .
$$

5. Finally, an explicit class of solutions to GW system (1.3) admitting two arbitrary real-valued functions of one real variable can be constructed based on the idea of Proposition 4. Suppose that $h$ and $\chi$ are real-valued functions of one variable $z+\bar{z}$, then the following function

$$
\rho=e^{-i \chi(z+\bar{z})} \frac{c_{1} e^{h(z+\bar{z})}+c_{2} e^{-h(z+\bar{z})}}{\bar{c}_{1} e^{h(z+\bar{z})}+\bar{c}_{2} e^{-h(z+\bar{z})}}, \quad c_{1}, c_{2} \in \mathbb{C},
$$

satisfies the constraint (5.1) and system (3.4), provided that the condition $c_{1} \bar{c}_{2}-c_{2} \bar{c}_{1}=0$ on the complex constants $c_{1}$ and $c_{2}$ holds. Defining the quantities

$$
f^{( \pm)}=c_{1} e^{h(z+\bar{z})} \pm c_{2} e^{-h(z+\bar{z})}
$$

and using Proposition 2, we can write a general solution of the sigma model (3.4) as follows

$$
\rho=\exp \left[-i \sum_{j=1}^{N} \chi_{j}(z+\bar{z})\right] \prod_{i=1}^{N} \frac{f_{i}^{(+)}}{\bar{f}_{i}^{(+)}}
$$

So, we have

$$
\partial \rho=\left[-i \sum_{j=1}^{N} \chi_{j}^{\prime}+\sum_{k=1}^{N} \frac{f_{k}^{(-)} h_{k}^{\prime}}{f_{k}^{(+)}}-\sum_{k=1}^{N} \frac{\bar{f}_{k}^{(-)} h_{k}^{\prime}}{\bar{f}_{k}^{(+)}}\right] \rho
$$

where the prime represents differentiation with respect to the single function argument of $\chi$ and $h$. Substituting (5.21) and (5.22) into transformation (3.3), we determine explicitly the corresponding form of multi-soliton solution of GW system (1.3)

$$
\begin{aligned}
& \psi_{1}=\frac{\epsilon}{2}\left(\rho\left[i \sum_{j=1}^{N} \chi_{j}^{\prime}+\sum_{k=1}^{N} \frac{\bar{f}_{k}^{(-)} h_{k}^{\prime}}{\bar{f}_{k}^{(+)}}-\sum_{k=1}^{N} \frac{\bar{f}_{k}^{(-)} h_{k}^{\prime}}{\bar{f}_{k}^{(+)}}\right]\right)^{1 / 2}, \\
& \psi_{2}=\frac{\epsilon}{2}\left(\rho\left[-i \sum_{j=1}^{N} \chi_{j}^{\prime}+\sum_{k=1}^{N} \frac{f_{k}^{(-)} h_{k}^{\prime}}{f_{k}^{(+)}}-\sum_{k=1}^{N} \frac{\bar{f}_{k}^{(-)} h_{k}^{\prime}}{\bar{f}_{k}^{(+)}}\right]\right)^{1 / 2} .
\end{aligned}
$$


Note that solutions (5.23) do not admit any singularities when $c_{1} \neq c_{2}$.

6. Using Proposition 4, an interesting class of periodic nonsplitting solutions of (3.4) satisfying the algebraic constraint (5.1) has the form

$$
\rho=\exp \left(\sum_{j=1}^{N}\left(\cos \left(z-a_{j}\right)-\cos \left(\bar{z}-\bar{a}_{j}\right)\right)\right)
$$

The derivatives of $\rho$ are given by

$$
\partial \rho=-\sum_{j=1}^{N} \sin \left(z-a_{j}\right) \rho, \quad \bar{\partial} \rho=\sum_{j=1}^{N} \sin \left(\bar{z}-\bar{a}_{j}\right) \rho
$$

Then using (3.3), we can find that the solutions of GW system (1.3) are

$$
\psi_{1}=\frac{\epsilon}{2}\left(-\sum_{j=1}^{N} \sin \left(\bar{z}-\bar{a}_{j}\right) \rho\right)^{1 / 2}, \quad \psi_{2}=\frac{\epsilon}{2}\left(-\sum_{j=1}^{N} \sin \left(z-a_{j}\right) \rho\right)^{1 / 2}, \quad p=\frac{1}{2}\left|\sum_{j=1}^{N} \sin \left(z-a_{j}\right)\right| .
$$

Note that solutions (5.19) and (5.25) do not admit any singularities. When $N=1$, the associated constant mean curvature surface can be computed from relations (1.4)

$$
u\left(X_{1}+i X_{2}\right)=-i v, \quad v\left(X_{1}-i X_{2}\right)=i u, \quad u v=e^{2 X_{3}},
$$

where $u=\exp (\cos (z-a))$ and $v=\exp (\cos (\bar{z}-\bar{a}))$. The corresponding surface is a cylinder having $X_{3}$ as a symmetry axis since $X_{1}$ and $X_{2}$ satisfy $X_{1}^{2}+X_{2}^{2}=1$. Similar calculations to the one given by (5.24) can be performed when cos in (5.24) is replaced by trigonometric and hyperbolic functions sin and cosh or sinh, respectively. This procedure provides us with several classes of periodic solutions of GW system (1.3).

Now, one can apply Proposition 4 again to solutions of the form (5.24) of sigma model (3.4) by replacing the cos function by sin and taking products of these resulting solutions, namely (5.24) and the modified one.

$$
\begin{gathered}
\rho=\exp \left(\sum_{j=1}^{N}\left(\cos \left(z-a_{j}\right)-\cos \left(\bar{z}-\bar{a}_{j}\right)+\sin \left(z-a_{j}\right)-\sin \left(\bar{z}-\bar{a}_{j}\right)\right)\right. \\
=\exp \left(\sum _ { j = 1 } ^ { N } \left(-2 \sin \left(\frac{z+\bar{z}-a_{j}-\bar{a}_{j}}{2}\right) \sin \left(\frac{z-\bar{z}-a_{j}+\bar{a}_{j}}{2}\right)\right.\right. \\
\left.\left.+2 \cos \left(\frac{z+\bar{z}-a_{j}-\bar{a}_{j}}{2}\right) \sin \left(\frac{z-\bar{z}-a_{j}+\bar{a}_{j}}{2}\right)\right)\right) .
\end{gathered}
$$

The corresponding solution of GW system (1.3) has the form

$$
\psi_{1}=\frac{1}{2} \epsilon\left(\rho \sum_{j=1}^{N}\left(-\sin \left(\bar{z}-\bar{a}_{j}\right)+\cos \left(\bar{z}-\bar{a}_{j}\right)\right)\right)^{1 / 2}, \quad \psi_{2}=\frac{1}{2} \epsilon\left(\rho \sum_{j=1}^{N}\left(-\sin \left(z-a_{j}\right)+\cos \left(z-a_{j}\right)\right)\right)^{1 / 2} .
$$

Note that there exists a resemblance of function $\rho$ given by (5.26) to a Calogero-Sutherland type potential [35].

6 DIFFERENTIAL CONSTRAINTS AND SOLUTIONS OF THE GW SYSTEM. 
We discuss now the existence of certain classes of solutions to the GW system (1.3) which can be obtained by subjecting (1.3) to specific differential constraints which allows us to reduce the overdetermined system to a system admitting a first integral. This fact simplifies considerably the process of solving the initial system (1.3) and consequently, certain classes of nonsplitting solutions can be constructed.

First let us discuss the case when we append two differential constraints to WE system (1.3) of the form

$$
\psi_{1} \partial \bar{\psi}_{1}+\bar{\psi}_{2} \partial \psi_{2}=0, \quad \bar{\psi}_{1} \bar{\partial} \psi_{1}+\psi_{2} \bar{\partial} \bar{\psi}_{2}=0
$$

In terms of the complex functions $\psi_{i}$, it has been shown [13] that the reduction of the overdetermined system composed of (1.3) and (6.1) is equivalent to a linear decoupled system

$$
\bar{\partial} \partial \psi_{i}+p_{0}^{2} \psi_{i}=0, \quad i=1,2
$$

with the first integral of the motion

$$
\left|\psi_{1}\right|^{2}+\left|\psi_{2}\right|^{2}=p_{0} \in \mathbb{R}
$$

Indeed, making use of GW system (1.3) and conditions (6.1), we have

$$
\partial p=\psi_{1}\left(\partial \bar{\psi}_{1}\right)+\bar{\psi}_{2}\left(\partial \psi_{2}\right)=0, \quad \bar{\partial} p=\bar{\psi}_{1}\left(\bar{\partial} \psi_{1}\right)+\psi_{2}\left(\bar{\partial} \bar{\psi}_{2}\right)=0
$$

This means that $p$ is a real constant. The overdetermined system (1.3) and (6.1) admits a threedimensional symmetry group, namely, the similitude group $\operatorname{Sim}(2, \mathbb{C})$. Its Lie subalgebra of algebra (2.1) is spanned by $\left\{T_{1}, T_{2}, H\right\}$ and the corresponding invariant is given by (6.3).

Now let us express constraints (6.4) in terms of the function $\rho$. Taking into account (3.4), the differential constraints (6.4) become

$$
\frac{\partial p}{\bar{\psi}_{2}}=\rho \partial \bar{\psi}_{1}+\partial \psi_{2}=\frac{\epsilon}{2}(\partial \rho)^{-1 / 2} \partial^{2} \rho-\epsilon \frac{(\partial \rho)^{1 / 2}}{1+|\rho|^{2}}(\rho \partial \bar{\rho}+\bar{\rho} \partial \rho)+\epsilon \rho \frac{(\partial \rho)^{1 / 2}(\partial \bar{\rho})}{1+|\rho|^{2}}=0
$$

and its respective complex conjugate. As a result, the overdetermined system composed of equations (3.4) and (6.5) for the function $\rho$ take the form

$$
\begin{aligned}
& \bar{\partial} \partial \rho-\frac{2 \bar{\rho}}{1+|\rho|^{2}} \partial \rho \bar{\partial} \rho=0, \quad \partial \bar{\partial} \bar{\rho}-\frac{2 \rho}{1+|\rho|^{2}} \partial \bar{\rho} \bar{\partial} \bar{\rho}=0 \\
& \partial^{2} \rho-\frac{2 \bar{\rho}}{1+|\rho|^{2}}(\partial \rho)^{2}=0, \quad \bar{\partial}^{2} \bar{\rho}-\frac{2 \rho}{1+|\rho|^{2}}(\bar{\partial} \bar{\rho})^{2}=0 .
\end{aligned}
$$

We show now that if the current $J$ given by (3.9) is constant, then all second derivatives of $\rho$ are determined in terms of $\rho$ and their first derivatives. In fact, differentiating the current $J$ with respect to $\partial$ we get

$$
\left(1+|\rho|^{2}\right)\left(\partial \bar{\rho} \partial^{2} \rho+\partial \rho \partial^{2} \bar{\rho}\right)-2 \partial \rho \partial \bar{\rho}(\bar{\rho} \partial \rho+\rho \partial \bar{\rho})=0
$$

Taking into account the system (6.6), we obtain

$$
\partial^{2} \bar{\rho}-\frac{2 \rho}{1+|\rho|^{2}}(\partial \bar{\rho})^{2}=0, \quad \bar{\partial}^{2} \rho-\frac{2 \bar{\rho}}{1+|\rho|^{2}}(\bar{\partial} \rho)^{2}=0 .
$$

Hence, all second order derivatives of $\rho$ are known in terms of $\rho$ and its derivatives. Note that all compatibility conditions are identically satisfied whenever equations (3.4) hold. Moreover, the 
nonsplitting exponential class of solution (5.18) is a solution of the system (6.6) and (6.7). Thus, we can formulate the following,

PROPOSITION 5. The overdetermined system (6.6) and (6.7) admits a first integral of the form

$$
\frac{(\partial \rho \bar{\partial} \bar{\rho})^{1 / 2}}{1+|\rho|^{2}}=p_{0}, \quad p_{0} \in \mathbb{R}
$$

PROOF. Differentiating the left hand side of (6.8) with respect to $\partial$, we obtain the expression

$$
\partial\left(\frac{(\partial \rho \bar{\partial} \bar{\rho})^{1 / 2}}{1+|\rho|^{2}}\right)=\frac{(\partial \rho)^{-1 / 2}(\bar{\partial} \bar{\rho})^{1 / 2}}{2\left(1+|\rho|^{2}\right)}\left[(\bar{\partial} \bar{\rho}) \partial^{2} \rho+\partial \rho(\partial \bar{\partial} \bar{\rho})-2 \frac{(\partial \rho)(\bar{\partial} \bar{\rho})}{1+|\rho|^{2}}(\partial \rho \bar{\rho}+\rho \partial \bar{\rho})\right] .
$$

Substituting (6.6) and (6.7) into system (6.9), this is identically zero, since

$$
\frac{2 \bar{\rho}}{1+|\rho|^{2}}(\bar{\partial} \bar{\rho})(\partial \rho)^{2}+\frac{2 \rho}{1+|\rho|^{2}} \partial \rho \partial \bar{\rho} \bar{\partial} \bar{\rho}-\frac{2 \bar{\rho}}{1+|\rho|^{2}} \bar{\partial} \bar{\rho}(\partial \rho)^{2}-\frac{2 \rho}{1+|\rho|^{2}} \partial \rho \partial \bar{\rho} \bar{\partial} \bar{\rho} \equiv 0 .
$$

Similarly, differentiating the left hand side of (6.8) with respect to $\bar{\partial}$ and making use of (6.6) and (6.7), we obtain that the resulting equation is identically satisfied. QED.

PROPOSITION 6. If the functions $\psi_{1}$ and $\psi_{2}$ satisfy the overdetermined system composed of the initial value problem for WE system (1.3) with $\psi_{i}(0)=0$ and differential constraints (6.1),

$$
\begin{gathered}
\partial \psi_{1}=p \psi_{2}, \quad \bar{\partial} \psi_{2}=-p \psi_{1}, \quad \psi_{i}(0)=0, \\
\psi_{1} \partial \bar{\psi}_{1}+\bar{\psi}_{2} \partial \psi_{2}=0, \quad \bar{\psi}_{1} \bar{\partial} \psi_{1}+\psi_{2} \bar{\partial} \bar{\psi}_{2}=0,
\end{gathered}
$$

then the general solution has the form,

$$
\psi_{i}=J_{0}\left(p_{0} \sqrt{\left(z-z_{i}^{1}\right)\left(\bar{z}-\bar{z}_{i}^{2}\right)}\right), \quad z_{i}^{1}, z_{i}^{2} \in \mathbb{C}, \quad i=1,2 .
$$

where $J_{0}$ is a Bessel function of order zero. In terms of the complex function $\rho$, which appears in system (6.6), the general solution has the form

$$
\rho=\frac{\psi_{1}}{\bar{\psi}_{2}}=\frac{J_{0}\left(p_{0} \sqrt{\left(z-z_{1}^{1}\right)\left(\bar{z}-\bar{z}_{1}^{2}\right)}\right)}{J_{0}\left(p_{0} \sqrt{\left(z-z_{2}^{1}\right)\left(\bar{z}-\bar{z}_{2}^{2}\right)}\right)}
$$

PROOF. If the function $W$ is a solution of the second order differential equation

$$
s_{i} W^{(2)}\left(s_{i}\right)+W^{(1)}\left(s_{i}\right)+p_{0}^{2} W\left(s_{i}\right)=0,
$$

where the differentiation of $W$ is with respect to $s_{i}$, then the general solution of the system (6.2) has the form

$$
\psi_{i}=W\left(s_{i}\right)
$$

where

$$
s_{i}=\frac{1}{4 p_{0}^{2}}\left(z-z_{i}^{1}\right)\left(\bar{z}-\bar{z}_{i}^{2}\right), \quad z_{i}^{1}, z_{i}^{2} \in \mathbb{C} .
$$

After a change of variable $s_{i}=\rho_{i}^{2}$, the differential equation (6.13) is reduced to the Bessel equation

$$
\rho_{i}^{2} J^{\prime \prime}\left(\rho_{i}\right)+\rho_{i} J^{\prime}\left(\rho_{i}\right)+4 p_{0}^{2} \rho_{i}^{2} J\left(\rho_{i}\right)=0
$$


and the general solution is given by

$$
\psi_{i}=J_{0}\left(p_{0} \sqrt{s_{i}}\right)=\sum_{k=0}^{\infty} \frac{(-1)^{k}}{k !^{2}}\left(p_{0}^{2 k}\left(z-z_{i}^{1}\right)^{k}\left(\bar{z}-\bar{z}_{i}^{2}\right)^{k}\right) .
$$

and hence (6.10) holds. Then using the definition (3.1) for the function $\rho$, we find that the general solution of the overdetermined system (6.6) and (6.7) is given by (6.12). QED.

Finally, let us discuss the case when the GW system is subjected to a single constraint

$$
\psi_{1} \partial \bar{\psi}_{1}+\bar{\psi}_{2} \partial \psi_{2}-\epsilon\left(\bar{\psi}_{1} \bar{\partial} \psi_{1}+\psi_{2} \bar{\partial} \bar{\psi}_{2}\right)=0, \quad \epsilon= \pm 1 .
$$

This is a weaker condition than (6.1), since two constraints have been combined. In terms of the complex function $\rho$, equation (6.14) becomes

$$
\left(\partial^{2} \rho-\frac{2 \bar{\rho}}{1+|\rho|^{2}}(\partial \rho)^{2}\right) \bar{\partial} \bar{\rho}-\epsilon\left(\bar{\partial}^{2} \bar{\rho}-\frac{2 \rho}{1+|\rho|^{2}}(\bar{\partial} \bar{\rho})^{2}\right) \partial \rho=0 .
$$

Note that if the function $\rho$ satisfies the overdetermined system composed of equations (3.4) and (6.15), then there exists a conserved quantity

$$
\frac{(\partial \rho)^{1 / 2}(\bar{\partial} \bar{\rho})^{1 / 2}}{1+|\rho|^{2}}=p(z+\epsilon \bar{z}) .
$$

In fact, from equations (6.16) and taking into account equations (3.4) and (6.15), we obtain

$$
(\partial-\epsilon \bar{\partial})\left(\frac{(\partial \rho)^{1 / 2}(\bar{\partial} \bar{\rho})^{1 / 2}}{1+|\rho|^{2}}\right)=p\left\{\frac{\partial^{2} \rho}{2 \partial \rho}-\frac{\bar{\rho} \partial \rho}{1+|\rho|^{2}}-\epsilon\left(\frac{\bar{\partial}^{2} \bar{\rho}}{2 \bar{\partial} \bar{\rho}}-\frac{\rho \bar{\partial} \bar{\rho}}{1+|\rho|^{2}}\right)\right\} \equiv 0 .
$$

Hence, the quantity $p$ is a real valued function of the argument $s=z+\epsilon \bar{z}$. This means that the solution of the system (1.3) and (6.14) is invariant under a two-dimensional symmetry algebra $\left\{T_{1}+\epsilon T_{2}, H\right\}$. This class of solutions have been discussed in detail in [27].

7 THE COMPLEX LAPLACE AND EIKONAL DIFFERENTIAL CONSTRAINTS.

Now, using transformation (3.3) and subjecting equations (3.4) to the differential constraints composed of the complex eikonal equation and Laplace equation, we show how to find, by integrating a certain ODE, a two-parameter family of solutions of the GW system. It shows that in the case of GW system (1.3) the proposed method for constructing solutions based on sigma model (3.4) can provide classes of solutions in addition to the ones already obtained in Sections 4 and 5 .

Consider the second order system of PDEs (3.4) for the functions $\rho$ and $\bar{\rho}$. If the gradients $\partial \rho \neq 0$ and $\bar{\partial} \rho \neq 0$ hold then, from equation (3.4i), we can find

$$
\bar{\rho}=\frac{\bar{\partial} \partial \rho}{2 \partial \rho \bar{\partial} \rho-\rho \bar{\partial} \partial \rho} .
$$

Eliminating $\bar{\rho}$ from equation (3.4ii), we obtain a fourth order PDE for the function $\rho$ in the form

$$
\bar{\partial} \partial\left(\frac{\bar{\partial} \partial \rho}{2 \partial \rho \bar{\partial} \rho-\rho \bar{\partial} \partial \rho}\right)-\frac{\rho(2 \partial \rho \bar{\partial} \rho-\rho \bar{\partial} \partial \rho)}{\partial \rho \bar{\partial} \rho} \partial\left(\frac{\bar{\partial} \partial \rho}{2 \partial \rho \bar{\partial} \rho-\rho \bar{\partial} \partial \rho}\right) \bar{\partial}\left(\frac{\bar{\partial} \partial \rho}{2 \partial \rho \bar{\partial} \rho-\rho \bar{\partial} \partial \rho}\right)=0 .
$$

First, let us now investigate a certain class of solution of (7.2) for which the scalar product of the gradient of $\rho$, denoted by $(\partial \rho \mid \bar{\partial} \rho)$, and the Laplace operator of $\rho$ namely $\bar{\partial} \partial \rho$ are constant on each level of the function $\rho$, that is,

$$
\begin{gathered}
(i) \quad(\partial \rho \mid \bar{\partial} \rho)=a(\rho), \\
(i i) \quad \bar{\partial} \partial \rho=b(\rho) .
\end{gathered}
$$


Here $a(\rho)$ and $b(\rho)$ are some complex-valued functions of $\rho$ of class $C^{1}$. System $(7.3 \mathrm{i})$ is called a complex eikonal system $[\mathbf{3 6 , 3 7 ]}$. Systems of PDEs similar to the type in (7.3) in n-dimensional real and complex vector spaces, with scalar product $(\nabla \rho \mid \nabla \rho)=a(\rho)$ with arbitrary signature $(p \neq 0, n-p)$, were investigated by geometrical methods for some classes of functions $a$ and $b$ [38-41]. Note that system (7.3) is invariant under the conformal group $\operatorname{Conf}(2, \mathbb{C})$ in general [39]. However, the overdetermined system (7.2) together with (7.3) is invariant under the similitude group $\operatorname{Sim}(2, \mathbb{C})$ smaller than $\operatorname{Conf}(2, \mathbb{C})$, and specific classes of solutions can be constructed.

We will now find all solutions $\rho$ of the system (7.3). In the case when $a=0$ and $b=0$, the solution of the problem (7.3) is obvious. The function $\rho$ has to be a holomorphic or antiholomorphic function. In the case when $a=0$ and $b$ is a nonzero arbitrary function, it turns out that system (7.3) does not possess a solution at all. In fact, if $a=0$ as in the previous case, this implies that $\rho$ is a holomorphic or antiholomorphic function which is in contradiction with the second condition (7.3 ii), since $\bar{\partial} \partial \rho=0$ holds and requires $b \equiv 0$. If $a=1$ and $b=0$ in equation (7.3), then the general solution of (7.3) is translationally invariant and given by

$$
u=\lambda z+\bar{\lambda} \bar{z}+u_{0}, \quad|\lambda|^{2}=1, \quad \lambda, u_{0} \in \mathbb{C} .
$$

Now let us discuss the complete set of solutions of the overdetermined system (7.3) in the complex domain when $a(\rho) \neq 0$ and $b$ is an arbitrary function of $\rho$. In this case, after a change of variable $\rho \rightarrow \gamma=f(\rho)$, with $f^{\prime}(\rho)=|\alpha(\rho)|^{-1 / 2}$, we may assume without loss of generality, that $a(\rho)=1$. Thus we consider the system of equations

$$
(\partial \rho \mid \bar{\partial} \rho)=1, \quad \bar{\partial} \partial \rho=b(\rho)
$$

Now, we will find the restriction on the function $b(\rho)$ such that there exist solutions of this system (7.5). More precisely, we have

PROPOSITION 7. For any complex-valued function $\rho$ defined in some neighbourhood of the point $z_{0} \in \mathbb{C}$, the following conditions are equivalent:

(a)

$$
(\partial \rho \mid \bar{\partial} \rho)=1, \quad \bar{\partial} \partial \rho=b(\rho)
$$

for a certain function $b(\rho) \neq 0$,

(b)

$$
(\partial \rho \mid \bar{\partial} \rho)=1, \quad \bar{\partial} \partial \rho=\frac{1}{\rho},
$$

(c) the function $\rho$ can be defined explicitly in the form

$$
\rho=\epsilon\left(4|z|^{2}+c_{1} z+c_{2} \bar{z}+\frac{1}{4} c_{1} c_{2}\right)^{1 / 2}, \quad \epsilon= \pm 1
$$

where $c_{1}$ and $c_{2}$ are arbitrary complex constants.

PROOF. First, we demonstrate that (a) implies (b). In fact, differentiating (7.5) with respect to $\partial$ and $\bar{\partial}$ respectively, and using (7.5), we get the following formulas

$$
\begin{aligned}
& \partial^{2} \rho=-b(\rho) \frac{\partial \rho}{\bar{\partial} \rho}, \quad \bar{\partial} \partial \rho=b(\rho), \\
& \bar{\partial}^{2} \rho=-b(\rho) \frac{\bar{\partial} \rho}{\partial \rho}, \quad \partial \rho \bar{\partial} \rho=1 .
\end{aligned}
$$


From the third order compatibility condition for the function $\rho$ we obtain,

$$
\begin{array}{ll}
\bar{\partial}\left(\partial^{2} \rho\right)=-\left(\dot{b}+2 b^{2}\right) \partial \rho, & \partial(\bar{\partial} \partial \rho)=\dot{b} \partial \rho, \\
\partial\left(\bar{\partial}^{2} \rho\right)=-\left(\dot{b}+2 b^{2}\right) \bar{\partial} \rho, & \bar{\partial}(\bar{\partial} \partial \rho)=\dot{b} \bar{\partial} \rho,
\end{array}
$$

where we denote by $\dot{b}=d b / d \rho$. Thus we have

$$
\dot{b}+b^{2}=0
$$

with the general integral

$$
b=\frac{1}{\rho-\rho_{0}}, \quad \rho_{0} \in \mathbb{C} .
$$

Without loss of generality, the constant $\rho_{0}$ can be absorbed in $\rho$, since the equations (7.6) are translationally invariant. Hence, (7.6) holds. Thus it remains to prove that (b) implies (c). It follows that if $|\rho| \neq 0$, (otherwise from (3.3) the functions $\psi_{i} \equiv 0$ ), then under the change of variable

$$
\rho=e^{v}
$$

the system (7.7) becomes

$$
\partial v \bar{\partial} v=e^{-2 v}, \quad \bar{\partial} \partial v=0 .
$$

Therefore, we get,

$$
\rho^{2}=e^{2 v}=e^{2(f+g)}
$$

such that

$$
e^{2 f}=2 \lambda z+c_{2}, \quad e^{2 g}=2 \bar{\lambda} \bar{z}+c_{1}, \quad|\lambda|^{2}=1,
$$

where $c_{1}, c_{2}$ and $\lambda$ are complex constants. Thus, by virtue of (7.13), the function $\rho$ takes the form

$$
\rho^{2}=4|z|^{2}+c_{1} z+c_{2} \bar{z}+\frac{1}{4} c_{1} c_{2}
$$

so (7.7) holds.

It is a rather surprising fact that from equation (7.2), the conditions (7.5) imply the algebraic constraint (5.1). However, condition (5.1) is inconsistent with the solution (7.7) of the system (7.5). Consequently, assumption (7.3) is a condition which is too strong to solve equation (7.2).

Finally, let us discuss the case when the GW system is subjected to some weaker condition than (7.3). Let us assume that there exists a function $u: \mathbb{C} \rightarrow \mathbb{C}$ which satisfies the overdetermined system (7.6) composed of the complex eikonal equation and Laplace equation

$$
(\partial u \mid \bar{\partial} u)=1, \quad \bar{\partial} \partial u=\frac{1}{u}
$$

Let the function

$$
\rho=y(u)
$$

be an arbitrary transformation of class $C^{4}$ of the dependent variable $u$. Obviously, in this case the function $\bar{\rho}$ given by (7.1) can also be expressed in terms of $y$ as follows

$$
\bar{\rho}=\frac{y^{(2)}+\frac{1}{u} y^{(1)}}{2 y^{(1) 2}-y\left(y^{(2)}+\frac{1}{u} y^{(1)}\right)} .
$$


We will demonstrate that the overdetermined system (7.2) and (7.14), under the transformation (7.15), admits a similarity form of solution. In fact, then the fourth order PDE (7.2) after substituting (7.15) with the help of system (7.14) reduces to the fourth order ODE for the function $y$, that is,

$$
u y^{(1) 2} y^{(4)}+2 y^{(1) 2} y^{(3)}-4 u y^{(1)} y^{(2)} y^{(3)}+3 u y^{(2) 3}-3 y^{(1)} y^{(2) 2}=0
$$

Putting

$$
w=y^{(1)}(u)
$$

we can reduce the order of (7.17) by one to give

$$
u w^{2} w^{(3)}+2\left(w^{2}-2 u w w^{(1)}\right) w^{(2)}+3 u w^{(1) 3}-3 w w^{(1) 2}=0 .
$$

Note that equation (7.19) passes the Painlevé test [23]. In fact, equation (7.19) can be integrated by using the similarity method. Introducing the new dependent variable

$$
w=u^{1 / n} v(u), \quad n \in \mathbb{Z},
$$

equation (7.19) becomes

$$
n u^{2} v^{2} v^{(3)}+v\left(2 n u v-4 n u^{2} v^{(1)}-v u\right) v^{(2)}-3 n u^{2}\left(v^{(1)}\right)^{3}+(1-3 n) u v v^{(1) 2}-v^{2} v^{(1)}=0 .
$$

The general integral of (7.21) takes the form

$$
v(u)=A u^{-(1+1 / n)} \cos ^{-2}(B \ln (u)+C),
$$

where $A, B, C$ are arbitrary complex constants. Substituting (7.22) into (7.20), we can find

$$
w=\frac{A}{u \cos ^{2}(B \ln (u)+C)} .
$$

Note that equation (7.19) can be integrated directly by introducing a new dependent variable of the form

$$
w=u^{r} v(u)
$$

for any complex $r$. The general integral of (7.19) has the same form as (7.23), since the factor $r$ is absorbed in the integration constants $A, B$ and $C$. Next, integrating (7.18), we obtain the function $\rho$ in terms of the variable $u$, we have

$$
\rho(u)=\frac{A}{B} \tan (B \ln (u)+C)+D, \quad D \in \mathbb{C} .
$$

On the other hand, from Proposition 5, the solution of system (7.7) has the form

$$
u=\epsilon\left(4|z|^{2}+c_{1} z+c_{2} \bar{z}+\frac{1}{4} c_{1} c_{2}\right)^{1 / 2}
$$

Thus, substituting (7.24) and (7.25), into PDE (7.2), we can generate a six-parameter family of periodic solutions of $(7.2)$

$$
\rho=\frac{A}{B} \tan \left(\frac{B}{2} \ln \left(4|z|^{2}+c_{1} z+c_{2} \bar{z}+\frac{1}{4} c_{1} c_{2}\right)+C\right)+D,
$$

Note that the solution (7.26) has singularities for values of $z$ which satisfy

$$
4|z|^{2}+c_{1} z+c_{2} \bar{z}+\frac{1}{4} c_{1} c_{2}=\exp \left(\frac{\pi}{B}(2 m+1)\right), \quad m \in \mathbb{Z}
$$


Substituting the function $\rho$ given by (7.26) into sigma model equation (3.4), we find additional constraints on the constants of integration $c_{1}=c_{2}=0,|A|= \pm B \in \mathbb{R}, C \in \mathbb{R}$ arbitrary and $D=0$. Then from (7.26), we get

$$
\rho=\frac{A}{|A|} \tan \left(\frac{|A|}{2} \ln \left(4|z|^{2}\right)+C\right) .
$$

Thus, we have proved the following Proposition:

PROPOSITION 8. If a complex-valued function $u$ defined on a certain open domain of $\mathbb{C}$ satisfies the system (7.14), then every function $\rho$ obtained from $u$ by transformation (7.15) of class

$C^{4}$ has a two-parameter family of solutions of the sigma model equation given by (7.27). The functions $\psi_{1}$ and $\psi_{2}$ defined by (3.3) in terms of the function $\rho$ are solutions of the GW system $(1.3)$,

$$
\begin{gathered}
\psi_{1}=\frac{\epsilon}{g(z, \bar{z})}\left(\frac{A}{|A|} \tan \left(\frac{|A|}{2} \ln \left(4|z|^{2}\right)+C\right)\right) \cdot\left[\frac{\bar{A}}{2 z} \sec ^{2}\left(\frac{|A|}{2} \ln \left(4|z|^{2}\right)+C\right)\right]^{1 / 2} \\
\psi_{2}=\frac{\epsilon}{g(z, \bar{z})}\left[\frac{A}{2 z} \sec ^{2}\left(\frac{|A|}{2} \ln \left(4|z|^{2}\right)+C\right)\right]^{1 / 2}
\end{gathered}
$$

where the function $g$ is defined by

$$
g(z, \bar{z})=1+|\rho|^{2}=1+\left(\frac{A}{|A|} \tan \left(\frac{|A|}{2} \ln \left(4|z|^{2}\right)+C\right)\right) \cdot\left(\frac{\bar{A}}{|A|} \tan \left(\frac{|A|}{2} \ln \left(4|z|^{2}\right)+C\right)\right) .
$$

Note that solution (7.28) is a periodic nonsingular solution for $|C|>0$ and can be interpreted as a kink type solution of GW system (1.3).

\section{APPLICATION TO CLASSICAL STRING THEORY.}

The study of stable classical configurations of strings is one of the most important problems of string theory $[\mathbf{6 , 1 2}]$. From a physical point of view, it has been proposed to describe QCD flux tubes, which are responsible for quark confinement using strings, and that QCD strings should take into account the extrinsic geometry of the string world sheet. In fact, two-dimensional nonlinear sigma models share many features with four-dimensional Yang-Mills theories.

A new approach to the classical configuration of strings in three-dimensional Euclidean space has been recently proposed [12]. This is based on the generalized Weierstrass formulas, which allow the construction of any surface in $\mathbb{R}^{3}$ starting with the system of two linear Dirac type equations

$$
\partial \psi_{1}=p \psi_{2}, \quad \bar{\partial} \psi_{2}=-p \psi_{1},
$$

where the function $p$ is a real valued function of $z$ and $\bar{z}$. In terms of the variables $p, \psi_{1}$ and $\psi_{2}$, the required action for the string has the following form,

$$
S=4 \mu_{0} \int\left(\left|\psi_{1}\right|^{2}+\left|\psi_{2}\right|^{2}\right)^{2} d x d y+\frac{4}{\alpha_{0}} \int p^{2} d x d y
$$

Classical configurations of strings can be described by common solutions of the Nambu-GotoPolyakov (NGP) action and the two linear equations provide surfaces in $\mathbb{R}^{3}$. If $v=\left|\psi_{1}\right|^{2}+\left|\psi_{2}\right|^{2}$ is the quantity that appears in the first fundamental form and $\varphi=v / p$, this equation takes the form,

$$
\partial \bar{\partial} \varphi+\left[2 p^{2}+\partial \bar{\partial} \ln p^{2}\right] \varphi-2 \alpha_{0} \mu_{0} p^{2} \varphi^{3}=0
$$

where $\alpha_{0}$ and $\mu_{0}$ appear in the NGP action above. The case of interest in this article is constant mean curvature, for which $\varphi=\varphi_{0}$ is constant. The equation in $\varphi$ above reduces to a second order linear PDE for $p$

$$
\partial \bar{\partial} \ln p^{2}+2\left(1-\alpha_{0} \mu_{0}\right) p^{2} \varphi_{0}^{2}=0
$$


Introducing the variable $\theta=\ln p^{2}$, this becomes the Liouville equation for the variable $\theta$

$$
\partial \bar{\partial} \theta+\beta e^{\theta}=0
$$

where $\beta=2\left(1-\alpha_{0} \mu_{0} \varphi_{0}^{2}\right)$. Since $\beta$ depends on the parameters $\alpha_{0}$ and $\mu_{0}$ which appear in (8.1), it is useful to substitute some of the solutions we have found to obtain values for $\beta$.

First of all, for the nonsplitting solutions presented in examples 1 to 5 in Section 5, we can calculate $p$ from the corresponding $\psi_{i}$ and it is found to satisfy (8.4) provided $\beta=0$. In fact, for any solutions which correspond to a $p$ of the factorized form $p=F(z) \bar{F}(\bar{z})$, it is a straightforward calculation to show that $\partial \bar{\partial} \theta=0$, and so (8.4) holds only for $\beta=0$. Some of the splitting solutions obtained by the authors in [13] yield nonzero values for $\beta$. Corresponding to the solutions of system (1.3) given by

$$
\psi_{1}=\epsilon n^{1 / 2} \frac{z^{n} \bar{z}^{(n-1) / 2}}{1+|z|^{2 n}}, \quad \psi_{2}=\epsilon n^{1 / 2} \frac{z^{(n-1) / 2}}{1+|z|^{2}},
$$

and the solutions of (1.3) given by the functions

$$
\psi_{1}=\epsilon \bar{\lambda}^{1 / 2} \frac{e^{\bar{\lambda} \bar{z} / 2}}{e^{-\lambda z}+e^{\lambda z}}, \quad \psi_{2}=\epsilon \lambda^{1 / 2} \frac{e^{-\lambda z / 2}}{e^{-\lambda z}+e^{\bar{\lambda} \bar{z}}},
$$

one finds that (8.4) is satisfied for both (8.5) and (8.6), provided that $\beta=2$. Finally, for the one soliton solutions of (1.3) given by

$$
\psi_{1}=\epsilon(a-b) \frac{z-a}{|z-a|^{2}+|z-b|^{2}}, \quad \psi_{2}=\epsilon(a-b) \frac{\bar{z}-b}{|z-a|^{2}+|z-b|^{2}},
$$

it is found that it will satisfy equation (8.4) provided that $\beta=2 /(a-b)^{2}$.

This shows that these specific solutions to GW system (1.3) can also be put into the WeierstrassNGP formulation of the classical theory of strings. In particular, the constraints we have obtained allow us to relate the constants in the NGP action in a specific way. With $\varphi_{0}=1$, the constants for the splitting solutions are related by $\alpha_{0} \mu_{0}=1$, and so the parameters $\alpha_{0}$ and $\mu_{0}$ would be restricted to this hyperbola. For the nonsplitting solutions, one would have for (8.6) the case $\alpha_{0} \mu_{0}=0$, which would imply that the second term in the action dominates the first term. For the case (8.7), the parameters satisfy $\alpha_{0} \mu_{0}=1-(a-b)^{-2}$, again restricted to a hyperbola.

\section{SUMMARY AND CONCLUDING REMARKS}

The main aim of the paper has been to provide a great variety of exact analytic solutions through the systematic use of the subgroup structure of the invariance group of the generalized Weierstrass system. We concentrated mainly on classical symmetries and as well on conditional symmetries for the GW system. The latter case refers to the symmetry of the overdetermined system obtained by supplementing the original system (1.3) with the differential constraints (6.1), and the original system (3.4) subjected to constraints (5.1) or (7.14). Thus, the solutions obtained for this overdetermined system are invariant under the action of the conditional symmetry algebras. They were investigated and a procedure for constructing them was proposed. We can summarize the results which were obtained using both symmetry methods in our work in the following cases:

1) Elementary solutions, that is, constant, algebraic with one or two simple poles, trigonometric and hyperbolic solutions.

2) Doubly periodic solutions which can be expressed in terms of Jacobi elliptic functions sn, cn and dn.

3) Some of the reduced equations can be written in terms of Painlevé transcendents. 
It is worth noting that the symmetry analysis of the sigma model (3.4) provides much larger classes of solutions to the original system (1.3) by means of transformation (3.3), than the symmetry analysis of GW system applied directly. This is due to the fact that there exists a gauge freedom in the definition of the function $\rho$, since the numerator and denominator of (3.1) can be multiplied by any complex function. Note also that the inverse mapping of (3.1) is a double valued function, and is provided by (3.3). We found that when, at least locally, the solutions of GW system (1.3) are single valued as a function of its complex independent variables, the system is a completely integrable one. Such a phenomena has been known since the the time of Z. Kovalevsky [42] in connection with the equations of the spinning top. In this case, the single-valued solutions of these equations are integrable, and wide classes of solutions have been constructed.

The construction of constant mean curvature surfaces which are embedded in three dimensional Euclidean space, by means of expression (1.4) is more difficult to integrate explicitly in the case when solutions of (1.3) are expressed in terms of Jacobi elliptic functions. In many of these cases, we deal with hyperelliptic integrals and the formulas obtained are quite complex expressions, and we skip them. However, the qualitative features, such as asymptotic behaviour, geometrical description, of these integrals can be investigated numerically (i.e. their singularity structure such as the existence of different types of poles, etc).

The question arises whether our approach can be extended to GW systems describing surfaces immersed in multi-dimensional Euclidean and pseudo-Riemannian spaces and if this may provide new classes of solutions. Recently, such generalization of the GW system has been achieved by B. Konopelchenko et al. [12,43-45] where, in particular, the explicit representations for generic surfaces conformally immersed into multi-dimensional Euclidean and pseudo-Euclidean spaces with different signatures have been derived.

In particular, the Dirac type system for the four complex-valued functions $\psi_{\alpha}$ and $\varphi_{\alpha}$ describing constant mean curvature surfaces immersed in four-dimensional Euclidean space is given by [44]

$$
\partial \psi_{\alpha}=e^{i \theta} p \varphi_{\alpha}, \quad \bar{\partial} \varphi_{\alpha}=-e^{-i \theta} p \psi_{\alpha}, \quad \alpha=1,2
$$

and their respective complex conjugate equations

$$
\bar{\partial} \bar{\psi}_{\alpha}=e^{-i \theta} p \bar{\varphi}_{\alpha}, \quad \partial \bar{\varphi}_{\alpha}=-e^{i \theta} p \bar{\psi}_{\alpha}
$$

where the following notation has been introduced

$$
p=\left(u_{1} u_{2}\right)^{1 / 2}, \quad u_{\alpha}=\left|\psi_{\alpha}\right|^{2}+\left|\varphi_{\alpha}\right|^{2}, \quad \alpha=1,2 .
$$

The function $\theta$ is assumed to be a real-valued function of $z$ and $\bar{z}$. It has been shown [44], that for each pair of complex fields $\left(\psi_{\alpha}, \varphi_{\alpha}\right)$ resulting from system (9.1), a set of constant mean curvature surfaces is obtained by means of the following parametrization

$$
(z, \bar{z}) \rightarrow r=\left(X_{1}(z, \bar{z}), \cdots, X_{4}(z, \bar{z})\right)
$$

such that

$$
\begin{aligned}
& X_{1}+i X_{2}=\int_{\Gamma}\left(\psi_{1} \psi_{2} d \bar{z}^{\prime}-\varphi_{1} \varphi_{2} d z^{\prime}\right), \\
& X_{1}-i X_{2}=\int_{\Gamma}\left(\bar{\psi}_{1} \bar{\psi}_{2} d z^{\prime}-\bar{\varphi}_{1} \bar{\varphi}_{2} d \bar{z}^{\prime}\right), \\
& X_{3}+i X_{4}=\int_{\Gamma}\left(\psi_{1} \bar{\varphi}_{2} d \bar{z}^{\prime}+\bar{\psi}_{2} \varphi_{1} d z^{\prime}\right),
\end{aligned}
$$




$$
X_{3}-i X_{4}=\int_{\Gamma}\left(\bar{\psi}_{1} \varphi_{2} d z^{\prime}+\psi_{2} \bar{\varphi}_{1} d \bar{z}^{\prime}\right)
$$

where $\Gamma$ is any contour in $\mathbb{C}$. The GW system (9.1) system possesses several conserved quantities, among them the following

(i) $\quad \partial\left(\psi_{\alpha} \psi_{\beta}\right)+\bar{\partial}\left(\varphi_{\alpha} \varphi_{\beta}\right)=0, \quad \bar{\partial}\left(\bar{\psi}_{\alpha} \bar{\psi}_{\beta}\right)+\partial\left(\bar{\varphi}_{\alpha} \bar{\varphi}_{\beta}\right)=0, \quad \alpha \neq \beta=1,2$,

(ii) $\quad \partial\left(\psi_{\alpha} \bar{\varphi}_{\beta}\right)-\bar{\partial}\left(\varphi_{\alpha} \bar{\psi}_{\beta}\right)=0, \quad \alpha \neq \beta=1,2$

(iii) $\quad \varphi_{\alpha} \partial \bar{\varphi}_{\alpha}+\bar{\psi}_{\alpha} \partial \psi_{\alpha}=0, \quad \bar{\varphi}_{\alpha} \bar{\partial} \varphi_{\alpha}+\psi_{\alpha} \bar{\partial} \bar{\psi}_{\alpha}=0, \quad \alpha=1,2$

(iv) $\quad \partial\left(\ln \psi_{\alpha}\right)\left(\bar{\partial} \ln \varphi_{\alpha}\right)=-p, \quad \bar{\partial}\left(\ln \bar{\psi}_{\alpha}\right)\left(\partial \ln \bar{\varphi}_{\alpha}\right)=-p, \quad \alpha=1,2$.

From equations (9.5 iv) and (9.1), we obtain the relation

$$
e^{i \theta}=-\left(\frac{\partial \psi_{\alpha}^{2}}{\bar{\partial} \varphi_{\alpha}^{2}}\right)^{1 / 2},
$$

and its reciprocal relation, which is given as follows

$$
e^{-i \theta}=i\left(\frac{\bar{\partial} \varphi_{\alpha}^{2}}{\partial \psi_{\alpha}^{2}}\right)^{1 / 2} .
$$

In particular, if the function $\theta$ is an entire function

$$
\bar{\partial} \theta=0
$$

then there exists the conservation of the current $J_{\alpha}$ defined as

$$
J_{\alpha}=\frac{\partial \psi_{\alpha}^{2}}{\bar{\partial} \varphi_{\alpha}^{2}}, \quad \bar{\partial} J_{\alpha}=0 .
$$

The existence of several conserved quantities is usually a strong indication that system (9.1) is an integrable one.

In the next stage of this research, using group theoretical techniques, the authors plan to generate in a systematic way large classes of solutions of GW system (9.1), which can be expressed as elementary and doubly periodic functions which are written in terms of the Jacobi elliptic functions. These solutions will lead to the construction of several classes of constant mean curvature surfaces by making use of equation (9.4) which can describe more diverse types of surfaces than the ones discussed in three dimensional Euclidean space.

\section{ACKNOWLEDGMENTS}

The authors thank Professor P. Winternitz, Université de Montréal, for helpful discussions on this topic. This work was supported by a research grant from NSERC of Canada and Fonds FCAR du Gouvernment du Québec. 


\section{APPENDIX A1.}

It is worth noting that the sigma model equations (3.4) are invariant under discrete transformations generated by

(i) the reflections

$$
\begin{aligned}
& Z_{1}: \quad z \rightarrow z, \quad \bar{z} \rightarrow-\bar{z}, \quad \rho \rightarrow \rho, \quad \bar{\rho} \rightarrow \bar{\rho} \\
& Z_{2}: \quad z \rightarrow z, \quad \bar{z} \rightarrow \bar{z}, \quad \rho \rightarrow-\rho, \quad \bar{\rho} \rightarrow-\bar{\rho} \\
& Z_{3}: \quad z \rightarrow-z, \quad \bar{z} \rightarrow \bar{z}, \quad \rho \rightarrow-\rho, \quad \bar{\rho} \rightarrow \bar{\rho}
\end{aligned}
$$

and their complex conjugates.

(ii) the inversion

$$
I: \quad z \rightarrow z, \quad \bar{z} \rightarrow \bar{z}, \quad \rho \rightarrow \frac{1}{\bar{\rho}}, \quad \bar{\rho} \rightarrow \frac{1}{\rho}
$$

(iii) the Caley transformation which maps the circle into the upper half plane

$$
C: \quad z \rightarrow z, \quad \bar{z} \rightarrow \bar{z}, \quad \rho \rightarrow \frac{1+i \eta}{1-i \eta}, \quad \bar{\rho} \rightarrow \frac{1-i \bar{\eta}}{1+i \bar{\eta}}
$$

(iv) the generalized $U(2)$ transformation for any $a, b \in \mathbb{C}$,

$$
U: \quad z \rightarrow z, \quad \bar{z} \rightarrow \bar{z}, \quad \rho \rightarrow \frac{a \eta+b}{-\bar{b} \eta+\bar{a}}, \quad \bar{\rho} \rightarrow \frac{\bar{a} \bar{\eta}+\bar{b}}{-b \bar{\eta}+a}, \quad a, b \in \mathbb{C}
$$

Note that the inversion transformation (iii) preserves the form of the sigma model equations (3.4), since substituting (A2) into (3.4 i), we get (3.4 ii). Clearly, applying this mapping a second time, we obtain $(3.4 \mathrm{i})$ again. This means that the square of the mapping has the same effect as the identity transformation. Note also that transformations (iii) and (iv) preserve the form of equations (3.4). Substituting (A3) or (A4) into the sigma model equations (3.4), we get

$$
\partial \bar{\partial} \rho-\frac{2 \bar{\rho}}{1+|\rho|^{2}} \partial \rho \bar{\partial} \rho=\frac{\eta+i}{(1+i \eta)^{3}\left(1+|\eta|^{2}\right)}\left(\partial \bar{\partial} \eta-\frac{2 \bar{\eta}}{1+|\eta|^{2}} \partial \eta \partial \bar{\eta}\right)=0
$$

and

$$
\partial \bar{\partial} \rho-\frac{2 \bar{\rho}}{1+|\rho|^{2}} \partial \rho \bar{\partial} \rho=\frac{\left(|a|^{2}+|b|^{2}\right)}{(\bar{b} \eta-\bar{a})^{2}}\left(\partial \bar{\partial} \eta-\frac{2 \bar{\eta}}{1+|\eta|^{2}} \partial \eta \bar{\partial} \eta\right)=0,
$$

respectively. The discrete subgroups were used to limit the range of parameters occuring in the classification list of one-dimensional subalgebras of the symmetry algebra of sigma model (3.4). 


\section{REFERENCES}

[1] Darboux G., Lecons sur systèmes orthogonaux et les coordonnes curvilignes, (Gauthier-Villars, Paris, 1910).

[2] Enneper, A., Analytisch-geometrische Untersuchungen., Nachr. Königl. Gesell. Wissensch. Georg-Augustus-Univ. Göttingen 1868, 12, 258-277. Osserman R., A Survey of Minimal Surfaces (Dover, New York, 1996).

[3] Konopelchenko B. G., Induced surfaces and their integrable dynamics, Stud. Appl. Math., 1996, 96, 9-51.

[4] Konopelchenko B. and Taimanov I., Constant mean curvature surfaces via an integrable dynamical system, J. Phys., 1996, A 29, 1261-1265.

[5] Gross D. G., Pope C. N., and Weinberg S., Two-dimensional Quantum Gravity and Random Surfaces (World Scientific, Singapore, 1992).

[6] Carrol R. and Konopelchenko B. G., Generalized Weierstrass-Enneper inducing, conformal immersions and gravity, Int. J. Modern Phys. A, 1996, 11, N7, 1183-1216.

[7] Viswanathan K. and Parthasarathy R., Ann. Phys. 1991, 206, 237, Phys. Rev. 1995, D 51, 5830 .

[8] Nelson D., Piran T. and Weinberg S., Statistical Mechanics of Membranes and Surfaces (World Scientific, Singapore, 1992).

[9] Amit D., Field Theory, the Renormalization Group and Critical Phenomena (McGraw-Hill, New York, 1978).

[10] Peradzynski Z., in Geometry and Nonlinear Partial Differential Equations in the Cauchy Problem for Dissipative Media, edited by W. Kosinski (Ossolineum, Wroclaw, 1979).

[11] Rozdestvenskii B. L. and Janenko N. N., Systems of Quasilinear Equations and their Applications to Gas Dynamics, Vol. 55 (AMS, Providence, RI, 1983).

[12] Konopelchenko B. G. and Landolfi G., On Classical String Configurations, Modern Phys. Letts. 1997, 12,40, 3161-3168. Konopelchenko B. G. and Landolfi G., Quantum effects for extrinsic geometry of strings via the generalized Weierstrass representation, Phys. Letts. 1998, B 444, 299-308. [13] Bracken P., Grundland A. M., Martina L., The Weierstrass-Enneper System for Constant Mean Curvature Surfaces and the Completely Integrable Sigma Model, J. Math. Phys, 1999, 40, 33793403.

[14] Ferapontov E. V. and Grundland A. M., Bäcklund links between different analytic descriptions of constant mean curvature surfaces, Journal of Nonlinear Mathematical Physics, 2000, 7,1, 14-21. [15] Bracken P. and Grundland A. M., On the Bäcklund transformation and the theorem of permutability for the generalized Weierstrass system, to appear in Inverse Problems.

[16] Olver P., Symmetry and explicit solutions of partial differential equations, Appl. Num. Math., 1992, 10, 307-324.

[17] Winternitz P., Lie groups and solutions of nonlinear partial differential equations, Integrable Systems, Quantum Groups and Quantum Field Theories, eds., L. A. Ibort and M. A. Rodriguez (Klewer, Dordrecht, 1993) pages 429-495.

[18] Olver P. J., Applications of Lie Groups to Differential Equations (Springer-Verlag, New York, 1993), 2nd Edition, Graduate Texts in Mathematics, vol. 107.

[19] Champagne B., Herman W., and Winternitz P., preprint CRM-1689, Montréal, 1990.

[20] Goursat E., Ann. Sci. Ec. Normale Sup., 1889, 3,6, 9.

[21] Patera J., Winternitz P., Sharp R., and Zassenhaus H., J. Math. Phys., 1977, 18, 2259.

[22] Rand D., Winternitz P., and Zassenhaus H., On the Identification of a Lie Algebra Given by its Structure Constants, I: Direct Decompositions, Levi Decompositions and Nilradicals, Lin. Alg. Appl., 1988, 109, 197-246. 
[23] Conte R., The Painlevé Approach to Nonlinear Ordinary Differential Equations, in The Painlevé Property, One Century Later, Chapter 3, editor R. Conte, (Springer, 1999).

[24] Fushchych W. I., Conditional sysmmetry of the equations of mathematical physics, Ukrain. Math. J., 1991, 43, 1456-1470.

[25] Grundland A. M., Martina L. and Rideau G., 1997 Partial differential equations with differential constraints in Lecture Notes AMS-CRM 11, Providence, 135-154.

[26] Kenmotsu K., Weierstrass formula for surfaces of prescribed mean curvature, Math. Ann. 1979, 245, 89-99.

[27] Bracken P. and Grundland A. M., On certain classes of solutions of the Weierstrass-Enneper system inducing constant mean curvature surfaces, J. Nonlin. Math. Physics, 1999 6,3, 294-313.

[28] Rand D., Winternitz P., and Zassenhaus H., Pascal Programs for Identification of Lie Algebras, II: SPLIT, A Program to Decompose Parameter-Free and Parameter-Dependent Lie Algebras into Direct Sums, Comp. Phys. Commun. 1987, 46, 297-309.

[29] Winternitz P., Comments on superposition rules for nonlinear coupled first-order differential equations, J. Math. Phys., 1984, 25, 2149-2153.

[30] Grundland A. M., Winternitz P. and Zakrzewski, J. Math. Phys., 1996, 37, 1501-1519.

[31] Painlevé P., Sur les equations différentialles du second ordre et d'ordre supérieur dont l'integrale générale est uniforme, 1 Mémoire, Acta Math., 1902, 25, 1.

[32] Gambier B., Sur les équations différentialles du second ordre et du premier degré dont l'integrale générale est a points critiques fixes, Acta Math., 1910, 33, 1.

[33] Ince E. L., Ordinary Differential Equations (Dover, New York, 1956).

[34] Byrd P. F. and Friedman M. D., Handbook of Elliptic Integrals for Engineers and Scientists (Springer, Berlin, 1971).

[35] Calogero F., Solution of the one-dimensional N-body problems with quadratic and/or inversely quadratic pair potentials, J. Math. Phys., 1971 12, 419-436.

[36] Collins C. B., All solutions to a nonlinear system of complex potential equations, J. Math. Phys., 1980, 21, 240-248.

[37] Collins C. B., Complex equations, special relativity and complex Minkowski space time, J. Math. Phys., 1980, 21, 249-255.

[38] Cieciura G. and Grundland A. M., A certain class of solutions of the nonlinear wave equation, J. Math. Phys., 1984, 25, 3460.

[39] Grundland A. M., Harnad J., and Winternitz P., Symmetry reduction for nonlinear relativistically invariant equations, J. Math. Phys., 1984, 25, 791-806.

[40] Basarab-Horwath P., Euler N., Euler M., and Fushchych W. I., Amplitude-phase representation for solutions of nonlinear d'Alembert equations, J. Phys., 1995, A 28, 6193-6201.

[41] Fushchych W. I., Shtelen W., and Serov M., Symmetry Analysis and Exact Solutions of Equations of Nonlinear Mathematical Physics, (Klewer, Dordrecht, 1993).

[42] Kovelevsky Z., Acta. Math., 1890, 14, 81.

[43] Konopelchenko B. G., Weierstrass representations for surfaces in 4D spaces and their integrable deformations via DS hierarchy, Preprint Math DG-9807129, 1998, University of Lecce.

[44] Konopelchenko B. G. and Landolfi G., Generalized Weierstrass representation for surfaces in multi-dimensional Riemann spaces, J. Geom. Phys., 1999, 29, 319-333.

[45] Konopelchenko B. G., and Landolfi G., Induced surfaces and their integrable dynamics. II Generalized Weierstrass representations in 4D spaces and deformations via DS hierarchy. Stud. Appl. Math. (to appear). 
Table 1: The Commutation Relations for the Lie Algebra $L(2.1)$

\begin{tabular}{|c|c|c|c|c|c|c|c|}
\hline & $K_{1}$ & $K_{2}$ & $D_{1}$ & $D_{2}$ & $T_{1}$ & $T_{2}$ & $H$ \\
\hline$K_{1}$ & 0 & 0 & $-K_{1}$ & 0 & $-2 D_{1}$ & 0 & 0 \\
$C_{2}$ & 0 & 0 & 0 & $-K_{2}$ & 0 & $-2 D_{2}$ & 0 \\
$D_{1}$ & $K_{1}$ & 0 & 0 & 0 & $-T_{1}$ & 0 & 0 \\
$D_{2}$ & 0 & $K_{2}$ & 0 & 0 & 0 & $-T_{2}$ & 0 \\
$T_{1}$ & $2 D_{1}$ & 0 & $T_{1}$ & 0 & 0 & 0 & 0 \\
$T_{2}$ & 0 & $2 D_{2}$ & 0 & $T_{2}$ & 0 & 0 & 0 \\
$H$ & 0 & 0 & 0 & 0 & 0 & 0 & 0 \\
\hline
\end{tabular}

Table 2: The one-dimensional subalgebras of (2.1) leading to a system of first order ODEs where $\alpha, \beta \in \mathbb{C}$.

\begin{tabular}{|c|c|c|c|c|}
\hline No. & Subalgebras & $\begin{array}{l}\text { Symmetry } \\
\text { Variable } \xi\end{array}$ & Orbits of the Group $G$ & Reduction to ODEs \\
\hline$L_{1}$ & $\begin{array}{c}D_{1}+\alpha D_{2}+\beta \\
\alpha \neq 0\end{array}$ & $\bar{z}^{-1} z^{\alpha}$ & $\begin{array}{l}\psi_{1}=A(\xi) z^{\beta-\alpha / 2}, \bar{\psi}_{1}=D(\xi) z^{-\beta+1 / 2} \\
\psi_{2}=B(\xi) z^{\beta-1 / 2}, \bar{\psi}_{2}=C(\xi) z^{-\beta+\alpha / 2}\end{array}$ & $\begin{array}{c}\alpha \xi \dot{A}+\left(\beta-\frac{\alpha}{2}\right) A \\
=(A D+B C) B \\
\xi^{2} \dot{B}=(A D+B C) A\end{array}$ \\
\hline$L_{2}$ & $T_{2}+D_{1}+\beta H$ & $z^{-1} e^{\bar{z}}$ & $\begin{array}{c}\psi_{1}=A(\xi) z^{\beta}, \bar{\psi}_{1}=B(\xi) z^{-(\beta+1 / 2)} \\
\psi_{2}=C(\xi) z^{\beta-1 / 2}, \bar{\psi}_{2}=D(\xi) z^{-\beta}\end{array}$ & $\begin{array}{c}-\xi \dot{A}+\beta A=(A B+C D) C \\
\xi \dot{C}=-(A B+C D) A\end{array}$ \\
\hline$L_{3}$ & $\begin{array}{c}T_{1}+T_{2}+\epsilon H \\
\epsilon= \pm 1\end{array}$ & $z-\bar{z}$ & $\begin{aligned} \psi_{1} & =A(\xi) e^{\epsilon z}, \bar{\psi}_{1}=\bar{A}(\bar{\xi}) e^{-\epsilon \bar{z}} \\
\psi_{2} & =B(\xi) e^{\epsilon z}, \bar{\psi}_{2}=\bar{B}(\bar{\xi}) e^{-\epsilon \bar{z}}\end{aligned}$ & $\begin{array}{c}\dot{A}+\epsilon A=C(A B+C D) e^{\epsilon \xi} \\
\dot{C}(\xi)=A(A B+C D) e^{\epsilon \xi}\end{array}$ \\
\hline$L_{4}$ & $D_{1}+\beta H$ & $\bar{z}$ & $\begin{array}{c}\psi_{1}=A(\xi) z^{\beta}, \psi_{1}=C(\xi) z^{-(\beta+1 / 2)} \\
\psi_{2}=D(\xi) z^{\beta-1 / 2}, \bar{\psi}_{2}=B(\xi) z^{-\beta}\end{array}$ & $\begin{array}{l}\beta A=D(A C+B D) \\
\dot{D}=-A(A C+B D)\end{array}$ \\
\hline$L_{5}$ & $\begin{array}{l}T_{1}+\epsilon H \\
\epsilon= \pm 1\end{array}$ & $\bar{z}$ & $\begin{array}{l}\psi_{1}=A_{0} e^{\epsilon(z-\bar{z})}, \psi_{1}=B_{0} e^{\epsilon(\bar{z}-z)} \\
\psi_{2}=C_{0} e^{\epsilon(z-\bar{z})}, \bar{\psi}_{2}=D_{0} e^{\epsilon(\bar{z}-z)}\end{array}$ & $\begin{array}{c}\epsilon A_{0}=C_{0}\left(A_{0} B_{0}+C_{0} D_{0}\right) \\
C_{0}=A_{0}\left(A_{0} B_{0}+C_{0} D_{0}\right)\end{array}$ \\
\hline$L_{6}$ & $D_{2}+\beta H$ & $z$ & $\begin{array}{c}\psi_{1}=A(\xi) \bar{z}^{\beta}, \bar{\psi}_{1}=C(\xi) \bar{z}^{-(\beta+1 / 2)} \\
\psi_{2}=D(\xi) \bar{z}^{\beta-1 / 2}, \bar{\psi}_{2}=B(\xi) \bar{z}^{-\beta}\end{array}$ & $\begin{aligned} \dot{A} & =B(A C+B D) \\
\beta B & =-A(A C+B D)\end{aligned}$ \\
\hline$L_{7}$ & $\begin{array}{l}T_{2}+\epsilon H \\
\epsilon= \pm 1\end{array}$ & $\begin{array}{l}z \\
z\end{array}$ & $\begin{array}{l}\psi_{1}=A_{0} e^{\epsilon(\bar{z}-z)}, \bar{\psi}_{1}=B_{0} e^{\epsilon(z-\bar{z})} \\
\psi_{2}=C_{0} e^{\epsilon(\bar{z}-z)}, \bar{\psi}_{2}=D_{0} e^{\epsilon(z-\bar{z})}\end{array}$ & $\begin{array}{l}\epsilon A_{0}=-C_{0}\left(A_{0} B_{0}+C_{0} D_{0}\right) \\
\epsilon C_{0}=-A_{0}\left(A_{0} B_{0}+C_{0} D_{0}\right)\end{array}$ \\
\hline
\end{tabular}

Table 3: The reflection subgroups admitted by system (4.6)

\begin{tabular}{|c|c|c|c|c|}
\hline 1 & $x \rightarrow-x$ & $y \rightarrow-y$ & $R \rightarrow R$ & $\phi \rightarrow \phi$ \\
2 & $x \rightarrow x$ & $y \rightarrow y$ & $R \rightarrow-R$ & $\phi \rightarrow-\phi$ \\
3 & $x \rightarrow-x$ & $y \rightarrow y$ & $R \rightarrow R$ & $\phi \rightarrow-\phi$ \\
& $x \rightarrow x$ & $y \rightarrow-y$ & $R \rightarrow R$ & $\phi \rightarrow-\phi$ \\
4 & $x \rightarrow-x$ & $y \rightarrow y$ & $R \rightarrow-R$ & $\phi \rightarrow \phi$ \\
& $x \rightarrow x$ & $y \rightarrow-y$ & $R \rightarrow-R$ & $\phi \rightarrow \phi$ \\
\hline
\end{tabular}




\begin{tabular}{|c|c|c|c|c|c|}
\hline 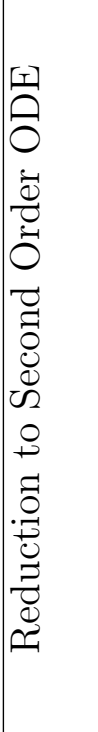 & 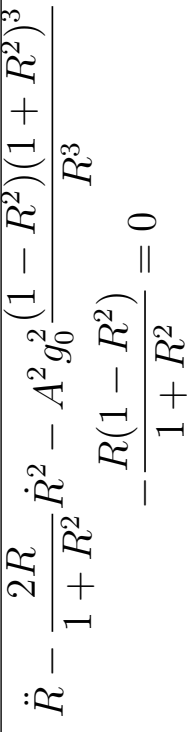 & 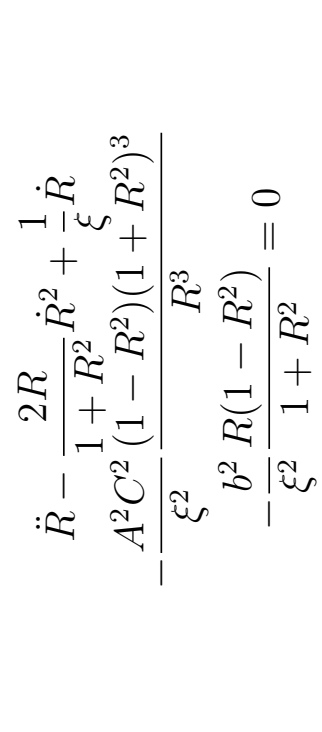 & 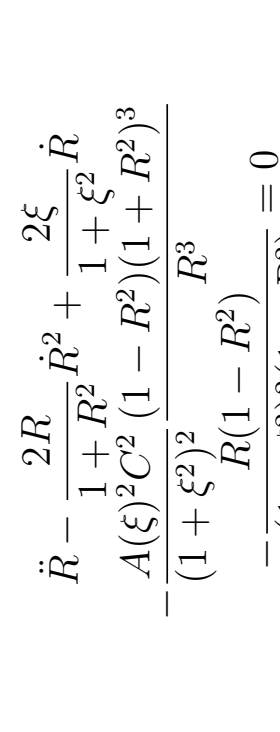 & 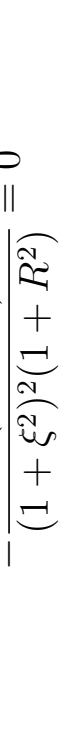 & 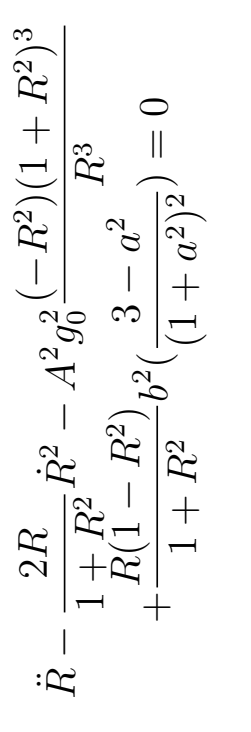 \\
\hline 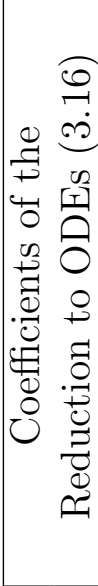 & 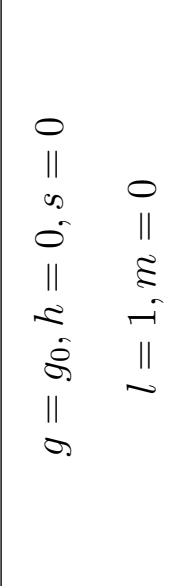 & 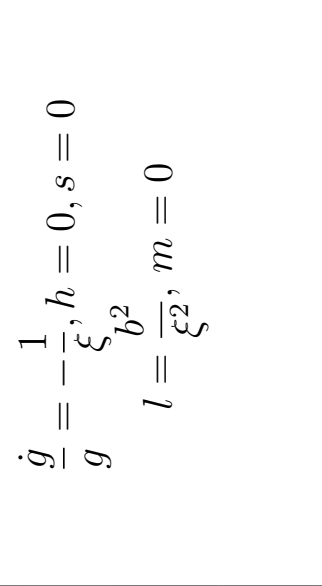 & 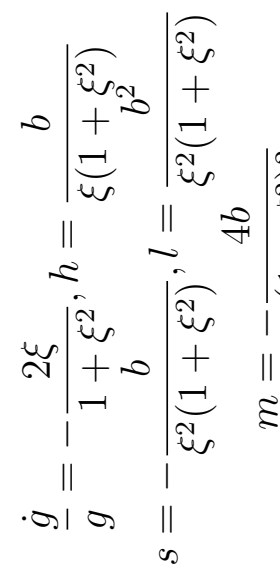 & 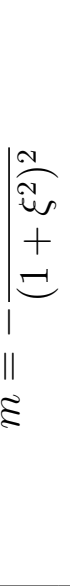 & 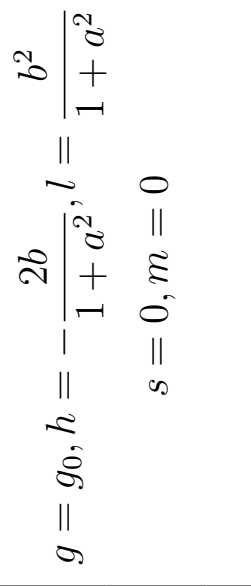 \\
\hline 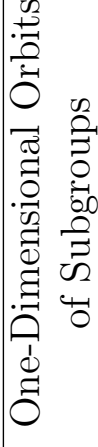 & 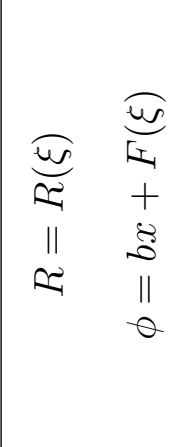 & 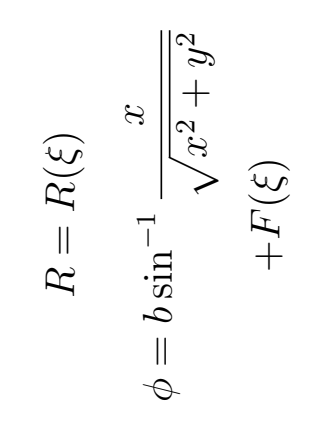 & 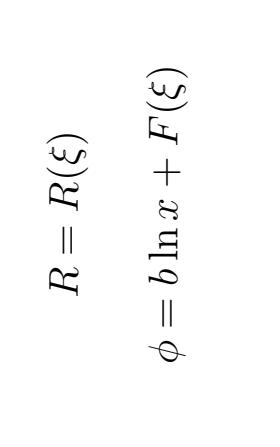 & & 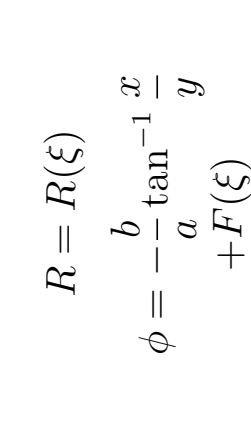 \\
\hline 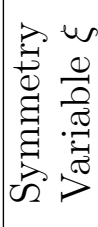 & $\lambda$ & 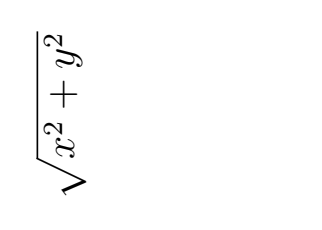 & ¿ & & 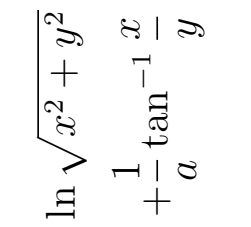 \\
\hline 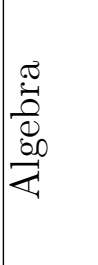 & $\begin{array}{l}\theta \\
0 \\
+ \\
\sigma^{-1}\end{array}$ & 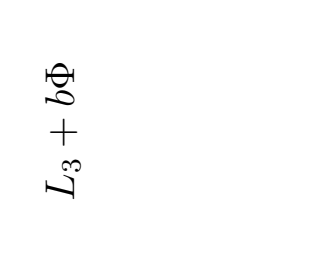 & $\begin{array}{l}\theta \\
\theta \\
+ \\
\theta\end{array}$ & & $\begin{array}{ll}0 & \\
0 & \\
+ & 0 \\
0 & 1 \\
0 & 0 \\
+ & 0 \\
0 & \end{array}$ \\
\hline$\stackrel{0}{z}$ & $\dot{\sim}$ & ৩ & $\therefore$ & & मं \\
\hline
\end{tabular}


Table 5: The Elliptic Solutions of the Sigma model (4.6), $\rho=R \exp (i \phi)$ with $R=\sqrt{-U(\eta)}$.

\begin{tabular}{|c|c|c|c|}
\hline No. & Order of Roots & Function $U(\eta)$ & Function $\beta$ Modulus $k$ \\
\hline 1. & $U_{1}>U_{2} \geq U>0$ & $U_{2} s n^{2}\left(\frac{\sqrt{4 K}}{\beta}\left(\eta-\eta_{0}\right), k\right)$ & $\beta=\frac{2}{\sqrt{U_{1}}} k^{2}=\frac{U_{2}}{U_{1}}$ \\
\hline 2. & $U_{1}>U_{2}>U \geq 0$ & $\frac{U_{1} U_{2}\left(1-s n^{2}\left(\frac{\sqrt{4 K}}{\beta}\left(\eta-\eta_{0}\right), k\right)\right)}{U_{1}-U_{2} s n^{2}\left(\frac{\sqrt{4 K}}{\beta}\left(\eta-\eta_{0}\right), k\right)}$ & $\beta=\frac{2}{\sqrt{U_{1}}}, k^{2}=\frac{U_{2}}{U_{1}}$ \\
\hline 3. & $U_{1} \geq U>U_{2}>0$ & $\frac{U_{1} U_{2}}{U_{1}}$ & $\beta=\frac{2}{\sqrt{U_{1}}}, k^{2}=\frac{U_{1}-U_{2}}{U_{1}}$ \\
\hline 4. & $U_{1}>U \geq U_{2}>0$ & $U_{1}-\left(U_{1}-U_{2}\right) s n^{2}\left(\frac{\sqrt{4 K}}{\beta}\left(\eta-\eta_{0}\right), k\right)$ & $\beta=\frac{2}{\sqrt{U_{1}}}, k^{2}=\frac{U_{1}-U_{2}}{U_{1}}$ \\
\hline 5. & $U>U_{1}>U_{2}>0$ & $-\frac{U_{1}-U_{2} s n^{2}\left(\frac{\sqrt{4 K}}{\beta}\left(\eta-\eta_{0}\right), k\right)}{s n^{2}\left(\frac{\sqrt{4 K}}{\beta}\left(\eta-\eta_{0}\right), k\right)-1}$ & $\beta=\frac{2}{\sqrt{U_{1}}}, k^{2}=\frac{U_{2}}{U_{1}}$ \\
\hline 6. & $\infty>U \geq U_{1}>U_{2}>0$ & $\frac{U_{1}}{s n^{2}\left(\frac{\sqrt{4 K}}{\beta}\left(\eta-\eta_{0}\right), k\right)}$ & $\beta=\frac{2}{\sqrt{U_{1}}}, k^{2}=\frac{U_{2}}{U_{1}}$ \\
\hline
\end{tabular}




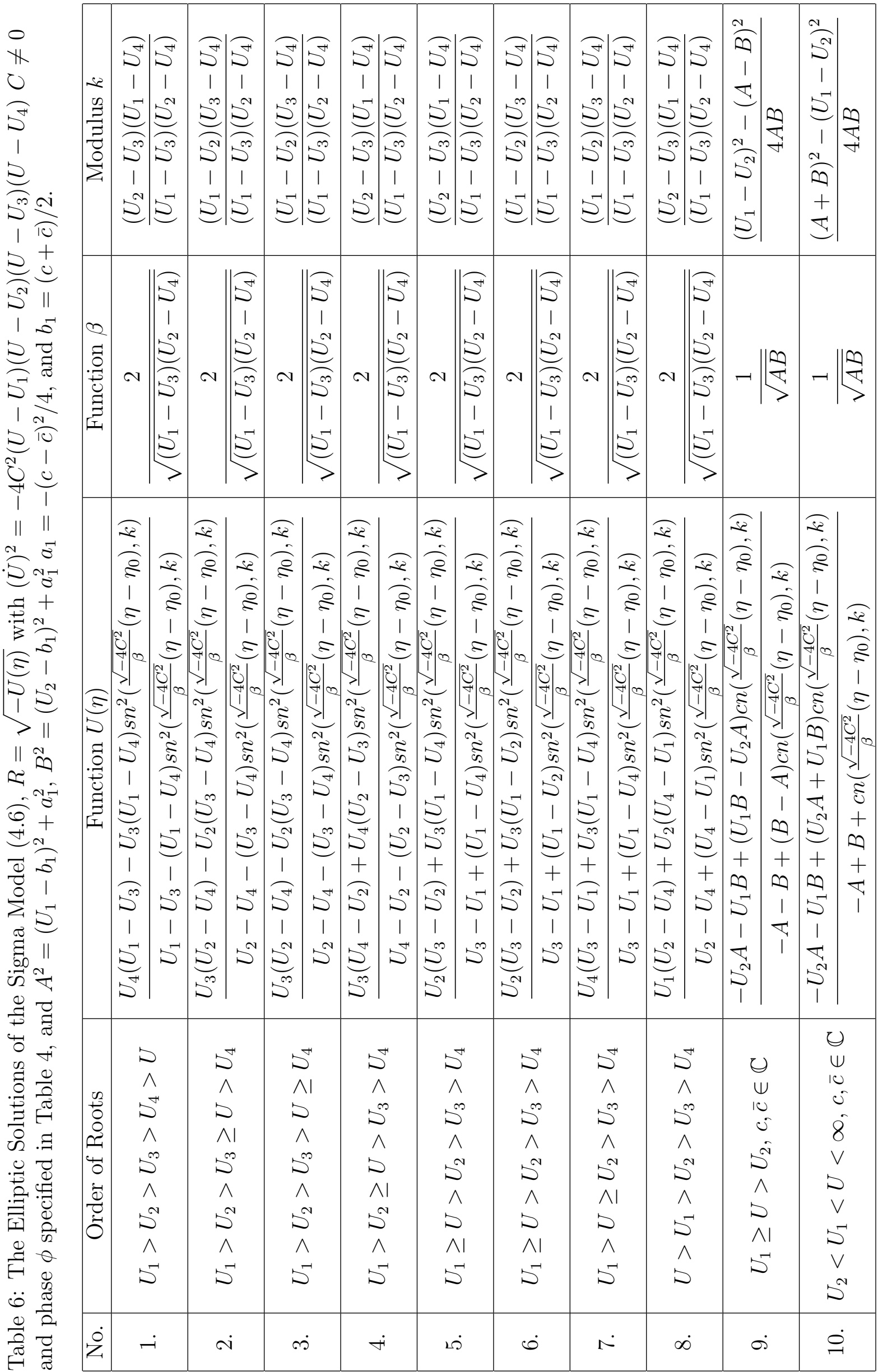




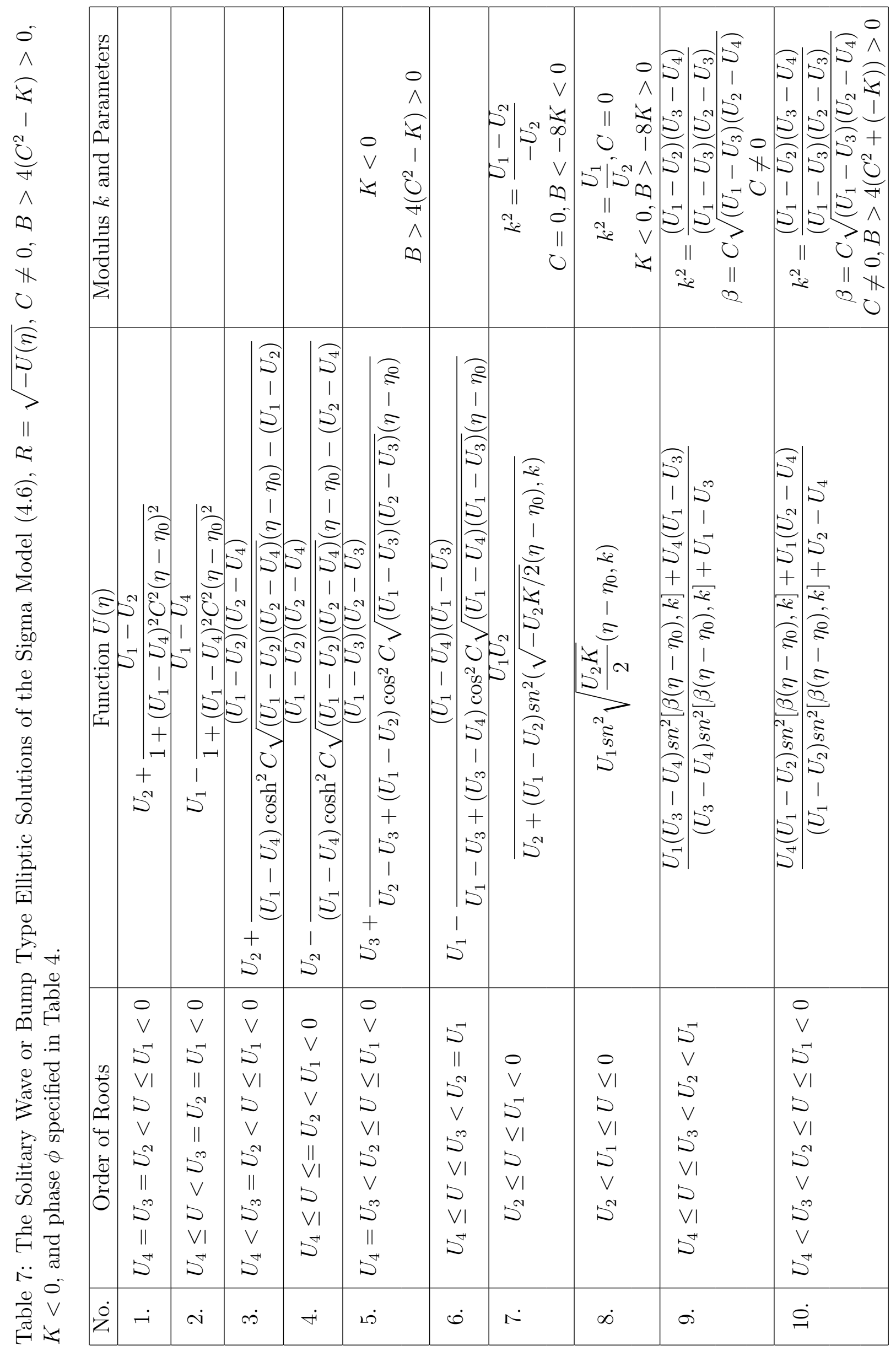

